\title{
STRATIFIABLE MAPS AND TOPOLOGICAL INVARIANTS
}

\author{
SYLVAIN E. CAPPELL AND JULIUS L. SHANESON
}

\section{Contents}

Introduction

1. Stratified pseudomanifolds

2. Self-dual complexes of sheaves

3. Proof of (2.1)

4. Stratified maps

5. Characteristic classes

6. Applications

6.1. Mismatch of dimensions

6.2. Resolutions, $L$-classes, and projective varieties

6.3. Discriminants

6.4. Higher signatures, symmetric signatures, and surgery obstructions

\section{INTRODUCTION}

Let $X$ and $Y$ be spaces and $f: X \rightarrow Y$ be a map between them. Then a large family of problems in topology (and geometry) involve the attempt to relate the invariants of $X$ and $Y$ via $f$. This attempt has been successful often for the case $X$ and $Y$ manifolds and $f$ the projection of a fibre bundle, for many types of important invariants. For example, the result of ChernHirzebruch-Serre [CHS] asserts that for $Y$ simply connected, the signature of $X$ is the product of the signatures of $Y$ and of the fibre $f^{-1}(y)$.

In this paper we undertake the study of maps in which the fiber may vary from point to point. The spaces $X$ and $Y$ will be any Whitney stratified spaces with even codimension strata (e.g., algebraic varieties) and $f$ will be a stratified map. This means essentially that for $V$ a component of a stratum of $Y$ (thus $V$ is an open manifold), $f^{-1} V$ is a union of strata of $X$ and the restriction of $f$ to $f^{-1} V$ is a locally trivial fibre bundle. For example, subanalytic maps of subanalytic sets can be stratified, with respect to Whitney

Received by the editors October 18, 1990.

1991 Mathematics Subject Classification. Primary 32C42, 55R55, 57R45, 14D99, 14F99, 14F45, $18 \mathrm{E} 30$.

Both authors were partially supported by NSF Grants, and the first author by a J. S. Guggenheim Fellowship (1989-1990). 
stratifications into subanalytic manifolds, and the same applies for complex analytic, real (or complex) semialgebraic, and complex algebraic maps [GM2, I.1.7]. In particular, even if $X$ and $Y$ are smooth manifolds, the class of maps under consideration is much broader than just bundle projections.

We will concentrate mainly on the characteristic $L$-classes

$$
L_{i}(X) \in H_{i}(X ; \mathbb{Q}) .
$$

For $X$ a closed manifold, $L_{i}(X)$ are the Poincare duals of Thom-Hirzebruch cohomology $L$-classes, which for smooth manifolds are in turn the $L$-polynomials in the Pontrjagin classes. For stratified spaces, these classes were defined by Goresky-MacPherson using their first definition of intersection homology in terms of p.l. chains [GM1]. (Alternate definitions can be found in [S], using the theory of "Witt spaces," and in [CSW], using self-dual complexes of sheaves; the topological invariance of these classes follows immediately from the latter. Cheeger defined these classes analytically on singular spaces with suitable metrics using his theory of $L^{2}$ differential forms.) A famous theorem of BrowderNovikov asserts that, for simply connected manifolds, the homotopy type and the $L$-classes determine the manifold up to a finite number of possibilities, and the variation of $L$-classes within a homotopy type can also be determined. (For example, a homotopy $C P^{3}$ is determined by its first Pontrjagin class, and it can vary by multiples of 24.) Recent work of Cappell-Weinberger shows that similar results hold for the isovariant classification of stratified spaces with simply connected strata and links.

Our methods can be applied to a range of topological invariants as well, e.g., discriminants, Novikov higher signatures, equivariant $K$-theory characteristic classes, etc. - see $\S 6$ and the end of this introduction for some examples. In a future work we will apply our methods to other (nontopological) invariants of stratified spaces with extra structure, e.g., Todd classes of algebraic varieties.

To describe the main result, let $f: X \rightarrow Y$ be a stratified map of compact even relative dimension of Whitney stratified spaces with only even codimension strata. Let $\mathscr{V}$ be the set of components of (open) strata of $Y$. Then each $V \in \mathscr{V}$ has a bundle neighborhood in $Y$ with fibre at $y \in V$ a stratified space $N(y)$, the "normal slice," with boundary $L(y)$, the "link." (In fact $N(y)$ is homeomorphic as a stratified space to the cone on $L(y)$.) Assume all strata and slices of $X$ and $Y$ have been compatibly oriented. For $y$ in a singular stratum, let

$$
E_{y}=f^{-1} N(y) / f^{-1} L(y) \cong f^{-1} N(y) \cup_{f^{-1} L(y)} c(L(y))
$$

be obtained from the inverse image of the normal slice by collapsing the boundary to a point, or equivalently, by attaching the cone on the boundary. For $y$ in the nonsingular stratum, let

$$
E_{y}=f^{-1}(y)
$$


be the "general fibre." Then $E_{y}$ is a stratified pseudomanifold with only even codimension strata, and hence has an index $\sigma\left(E_{y}\right)$, the signature of the intersection pairing on the middle-dimensional intersection homology with middle perversity. Let $j_{*}$ denote (generically) the map induced on homology by the inclusion of the closure $\bar{V}$ of $V \in \mathscr{V}$.

Theorem (see (5.8)). Assume that each $V \in \mathscr{V}$ is simply connected. Let $y_{V}$ be a chosen point of $V$. Then

$$
f_{*} L_{i}(X)=\sum_{\mathscr{V}} \sigma\left(E_{y_{V}}\right) j_{*} L_{i}(\bar{V}) .
$$

Thus for the signature $L_{0}(X)=\sigma(X)$, we obtain

Corollary. Under the same hypotheses,

$$
\sigma(X)=\sum_{\mathscr{V}} \sigma\left(E_{y_{V}}\right) \sigma(\bar{V}) .
$$

In the corollary, the contribution from the nonsingular part of $Y$ is the formula of [CHS], and the remaining terms describe the error in their formula when the map has singularities. Without the simple connectivity assumption, the terms on the right in these formulas must be replaced by twisted $L$-classes and twisted signatures (see (5.7)), and the singular contributions in the corollary describe the error in Atiyah's formula for signature of fibre bundles with nontrivial monodromy when the map has singularities.

The statement of the above result makes sense using only the intersection homology theory of [GM1], in terms of p.l. chains, at least for $X$ and $Y$ triangulated. In some cases the statement can even be formulated in terms of ordinary homology. However, the proof uses the sheaf-theoretic methods of [GM2, Bo], some of the foundational topological aspects of the theory of perverse sheaves [BBD, $\S \S 1,2]$, and a new notion of cobordism of self-dual complexes of sheaves (§2). (Note. We will always use the indexing conventions of [GM2].) A self-dual complex of sheaves on a stratified pseudomanifold $Y$ is a bounded constructible complex $\mathbb{S}^{\bullet}$ over $Y$ together with a duality isomorphism

$$
\mathbb{S}^{\bullet} \cong \mathfrak{D}\left(\mathbb{S}^{\bullet}\right)[\operatorname{dim} X]
$$

in the derived category $D^{b}(Y ; \mathfrak{K})$ over a field $\mathfrak{K}$. For compact even-dimensional spaces, a self-dual complex of sheaves $\mathbb{S}^{\bullet}$ has an induced nonsingular pairing $\mathbb{Q}_{\mathbb{S}^{\bullet}}$ on the middle-dimensional hypercohomology $\mathscr{H}^{-(\operatorname{dim} X) / 2}\left(X ; \mathbb{S}^{\bullet}\right)$ whose class in the Witt group over $\mathfrak{K}$ is invariant under cobordism.

Theorem (see (4.7)). Let $f: X^{p} \rightarrow Y^{n}$ be a stratified map of oriented Whitney stratified spaces. Assume $X$ is compact, $Y$ has only even codimension strata, and

$$
t=\frac{1}{2}(p-n)
$$


is an integer. Let $\mathbb{S}^{\bullet}$ be a self-dual complex of sheaves on $X$. Let $E_{y}$ be as above, $y \in V \in \mathscr{V}$. Let

$$
E_{y} \stackrel{i_{y}}{\longleftarrow} f^{-1} \stackrel{\circ}{N}(y) \stackrel{\rho_{y}}{\longrightarrow} X
$$

be inclusions. Let $Z_{y}=E_{y}-f^{-1} \stackrel{\circ}{N}(y)$. Then $\left(c=c(V)=\frac{1}{2} \operatorname{codim} V\right)$

(i) $\mathbb{S}^{\bullet}(y)=\tau_{\leq-c-t-1}^{Z_{y}} R i_{y_{*}} \rho_{y}^{!} \mathbb{S}^{\bullet}$ is a self-dual complex of sheaves on $E_{y}$, and

(ii) if $\mathfrak{H}_{f}^{V}\left(\mathbb{S}^{\bullet}\right)$ is the local system over $V$ with stalks

$$
\mathfrak{H}_{f}^{V}\left(\mathbb{S}^{\bullet}\right)_{y}=\mathscr{H}^{-c-t}\left(E_{y} ; \mathbb{S}^{\bullet}(y)\right)
$$

and with the induced nonsingular pairing $\mathscr{Q}_{\mathbb{S}^{*}(y)}$ on each stalk, then $f_{*} \mathbb{S}^{\bullet}[-t]$ is cobordant to

$$
\sum_{\mathscr{V}} j_{*}\left(\mathbb{C}_{\bar{m}}^{\bullet}\left(\bar{V} ; \mathfrak{H}_{f}^{V}\left(\mathbb{S}^{*}\right)\right)\right)[c(V)]
$$

This theorem can be interpreted in part as the statement that up to cobordism the Beilinson-Berstein-Deligne decomposition theorem holds in the topological category. In the case of a projective algebraic or analytic map, the above sum can be identified (nontrivially) with a piece of the BBD decomposition. Note that this theorem applies to any self-dual complex of sheaves, not just those "d'origine geometrique" and implies (for $f=\mathrm{id}$ ) that every self-dual complex is cobordant to a sum of intersection complexes. In the sequel, this last statement is proved first. It is then applied to $f_{*} \mathbb{S}^{\circ}[-t]$ and the individual terms are computed to be as in the preceding theorem.

For many important topological invariants, a decomposition up to cobordism is sufficient to provide exact formulae. For example, for $\mathbb{S}^{\bullet}=\mathbb{I C}_{\bar{m}}^{\bullet}(X)$ the complex of intersection chains (with coefficients in $\mathfrak{K}$ ), it is not hard to see that $\mathbb{S}^{\bullet}(y)=\mathbb{I} \mathbb{C}_{\bar{m}}^{\bullet}\left(E_{y}\right)$. Hence

Corollary. Let $f$ be above. Let $p$ be even. Then, in the Witt group over $\mathfrak{K}$,

$$
I H_{p / 2}^{\bar{m}}(X) \sim \sum_{\mathscr{V}} I H_{n / 2-c}^{\bar{m}}\left(\bar{V} ; I H_{c+t}^{\bar{m}}\left(E_{y_{V}}\right)\right) .
$$

This result implies the zero-dimensional case of the above formula for $L$ classes, i.e., the above corollary. In fact, every self-dual complex of sheaves has characteristic homology $L$-classes (see $\S 5$ ). These are cobordism invariants, and the formula for $L$-classes then follows from the last theorem above.

The results of this paper probably hold in a wider category of homotopy stratified maps of manifold homotopy stratified spaces.

In the final section we give some simple applications. More elaborate applications and examples will be discussed elsewhere.

We briefly mention an equivariant analogue of the results. Let the finite group $G$ act on the compact stratified space $X$ with even codimension strata. Then in [CSW] an equivariant characteristic class

$$
\Delta^{G}(X) \in K_{*}^{G}(X)
$$


in the equivariant $K$-homology of $X$ (with $\mathbb{Z}_{\text {(odd) }}$ coefficients) is defined. The image of this class in the representation ring $K_{*}^{G}(\mathrm{pt})$ is the $G$-signature $\sigma_{G}(X)$.

Let $f: X \rightarrow Y$ be a stratified map of Whitney stratified spaces, which is also equivariant with respect to actions of the finite group $G$ on $X$ and on $Y$. We assume the action preserves normal slices. (This hypothesis can be greatly weakened if one works in the wider category of homotopy stratified spaces and maps.) Then, with the above notation, $E_{y}$ has an action of the normalizer $N\left(G_{y}\right)$ of the isotropy subgroup

$$
G_{y}=\{g \mid g y=y\} .
$$

Theorem. Under an assumption of trivial monodromy (e.g., each stratum of $Y$ simply connected),

$$
f_{*} \Delta^{G}(X)=\sum_{\mathscr{V}} \sigma_{N\left(G_{y}\right)}\left(E_{y_{V}}\right) j_{*} \Delta^{G}(\bar{V}) .
$$

When $f$ is a bundle projection, this result is in [CSW].

Note that even in the case of the trivial group. $G=\{e\}$, this result provides a lifting of the first theorem above from homology characteristic classes over $\mathbb{Q}$ to $K$-homology classes over $\mathbb{Z}_{\text {(odd) }}$. Passage to homology for general finite $G$ gives a formula for the "Atiyah-Singer" equivariant classes. There is a twisted version of the result for nontrivial monodromy. Discussion in detail of the theorem and related questions in transformation groups will appear elsewhere.

\section{STRATIFIED PSEUdOMANIFOLDS}

A stratified pseudomanifold of dimension $n$ is defined by induction on dimension as a space, filtered by closed subspaces,

$$
\varnothing=Y_{-1} \subset Y_{0} \subset Y_{1} \subset \cdots \subset Y_{n-2}=Y_{n-1} \subset Y,
$$

such that the strata $Y_{i}-Y_{i-1}$ satisfy the following properties:

SP1. $Y-Y_{n-1}$ is an $n$-dimensional manifold and is dense in $X$.

SP2. $Y_{i}-Y_{i-1}$ is an $i$-dimensional manifold (or $\varnothing$ ).

SP3. For $y \in Y_{i}-Y_{i-1}$, there exists an open neighborhood $U$ of $x$ in $Y_{i}-Y_{i-1}$, a compact stratified pseudomanifold $L_{y}$ of dimension $n-i-1$, and a stratum-preserving homeomorphism

$$
\varphi_{y}: U \times c L_{y} \rightarrow Y .
$$

In general, we will use freely material from [GM2] and [Bo]. In particular for

$$
j_{y}:\{y\} \rightarrow Y
$$

the inclusion, we have (see [GM2, §2; GS, §6; Bo; CS2, 4.3])

(1.1) If $y \in Y_{m-k}-Y_{m-k-1}$ then

$$
H^{i}\left(j_{y}^{*} \mathbb{I} \mathbb{C}_{\bar{p}}^{\bullet}(Y ; \mathfrak{L})\right)= \begin{cases}I H_{-i-m+k-1}^{\bar{p}}\left(L_{y} ; \mathfrak{L}\right) & \text { for } i \leq p(k)-m, \\ 0 & \text { for } i>p(k)-m .\end{cases}
$$


(1.2) If $y \in Y_{m-k}-Y_{m-k-1}$ then

$$
H^{i}\left(j_{y}^{!} \mathbb{I} \mathbb{C}_{\bar{p}}^{\bullet}(Y ; \mathfrak{L})\right)= \begin{cases}I H_{-i}^{p}\left(L_{y} ; \mathfrak{L}\right) & \text { for } i>p(k)-k+1, \\ 0 & \text { for } i \leq p(k)-k+1 .\end{cases}
$$

(1.3) Let $\mathbb{A}^{\bullet}$ and $\mathbb{B}^{\bullet}$ be bounded complexes of sheaves over $R$ on the stratified pseudomanifold $Z^{m}$, constructible with respect to the stratification $\left\{Z_{k}\right\}$. Let $\bar{p}$ be a perversity and assume that for $z \in Z_{m-k}-Z_{m-k-1}, k \geq 2$,

(i) $H^{i}\left(j_{z}^{*} \mathbb{A}^{\bullet}\right)=0$ for $i>p(k)-m$.

Then restriction to $Z-Z_{m-2}=Z-\Sigma$ induces an injection

$$
\operatorname{Hom}_{D^{b}(Z)}\left(\mathbb{A}^{\bullet}, \mathbb{B}^{\bullet}\right) \rightarrow \operatorname{Hom}_{D^{b}(Z-\Sigma)}\left(\mathbb{A}^{\bullet}\left|(Z-\Sigma), \mathbb{B}^{\bullet}\right|(Z-\Sigma)\right)
$$

if

(ii) $H^{i}\left(j_{z}^{!} \mathbb{B}^{\bullet}\right)=0$ for $i \leq p(k)-k$

for $z$ as above, and an isomorphism if

(iii) $H^{i}\left(j_{z} ! \mathbb{B}^{\bullet}\right)=0$ for $i \leq p(k)-k+1$.

Further, if $\mathbb{H}^{i}\left(\mathbb{A}^{\bullet} \mid(Z-\Sigma)\right)=0$ for $i>s$ and $\mathbb{H}^{i}\left(\mathbb{B}^{\bullet} \mid(Z-\Sigma)\right)=0$ for $i<s$, there is an injection

$$
\operatorname{Hom}_{D^{b}(Z)}\left(\mathbb{A}^{\bullet}, \mathbb{B}^{\bullet}\right) \subset \operatorname{Hom}\left(\mathbb{H}^{s}\left(A^{\bullet} \mid(Z-\Sigma)\right), H^{s}\left(\mathbb{B}^{\bullet} \mid(Z-\Sigma)\right)\right)
$$

if (i) and (ii) hold, which is an isomorphism if (i) and (iii) hold.

The conditions (i) and (iii) are precisely the stalk and costalk conditions $[A X 1](c)$ and $[A X 1]\left(d^{\prime \prime}\right)$ of [GM2, 3.3], and the proof is readily extracted from the arguments of [GM2, 3.5]. See also [Bo]. In fact, let $U_{k}=Z-Z_{n-k}$, and let

$$
i_{k}: U_{k} \rightarrow U_{k+1}
$$

be the inclusion. Write $\mathbb{A}_{k}^{\bullet}=\mathbb{A}^{\bullet}\left|U_{k}, \mathbb{B}_{k}^{\bullet}=\mathbb{B}^{\bullet}\right| U_{k}$. By induction, assume the results hold over $U_{k}$. As in [GM2, 3.3], the weaker (stronger) costalk condition implies that the canonical adjunction map

$$
\mathbb{B}_{k+1}^{\bullet} \rightarrow R\left(i_{k}\right)_{*}\left(\mathbb{B}_{k}^{\bullet}\right)
$$

obviously a quasi-isomorphism over $U_{k}$, also induces isomorphisms of cohomology stalks over $U_{k+1}-U_{k}$ in dimensions at most $p(k)-m-1$ and a surjective map (isomorphism) in dimension $p(k)-m$. Also $\mathbb{H}^{i}\left(\mathbb{A}_{k+1}^{\bullet}\right)=0$ for $i>p(k)-m$ (since $p(k)$ is nondecreasing). From the argument used in the lifting result given in [GM2, 1.15], we then have that

$$
\operatorname{Hom}_{D^{b}\left(U_{k+1}\right)}\left(\mathbb{A}_{k+1}^{\bullet}, \mathbb{B}_{k+1}^{\bullet}\right)
$$

injects into (is isomorphic to)

$$
\begin{aligned}
\operatorname{Hom}_{D^{b}\left(U_{k+1}\right)}\left(\mathbb{A}_{k+1}^{\bullet}, R\left(i_{k}\right)_{*}\left(\mathbb{B}_{k}^{\bullet}\right)\right) & \cong \operatorname{Hom}_{D^{b}\left(U_{k+1}\right)}\left(R\left(i_{k}\right)_{*}\left(\mathbb{A}_{k}^{\bullet}\right), R\left(i_{k}\right)_{*}\left(\mathbb{B}_{k}^{\bullet}\right)\right) . \\
& \cong \operatorname{Hom}_{D^{b}\left(U_{k}\right)}\left(\mathbb{A}_{k}^{\bullet}, \mathbb{B}_{k}^{\bullet}\right),
\end{aligned}
$$


which by induction injects (is isomorphic) to $\operatorname{Hom}_{D^{b}\left(U_{2}\right)}\left(\mathbb{A}_{2}^{\bullet}, \mathbb{B}_{2}^{\bullet}\right)$. The final statement of (4.3) follows by applying the first proposition in [GM2, 1.15].

\section{Self-DuAl Complexes of Sheaves}

Let $Y^{m}$ be a stratified pseudomanifold. Let $\mathfrak{K}$ be a field. Throughout this section we assume that $Y$ is oriented and that the strata and links are also given orientations that are compatible under (SP3) with the orientation of $Y$.

By a self-dual complex of sheaves on $Y$, over $\mathfrak{K}$, we mean a pair $\left(\mathbb{S}^{\bullet}, d\right)$, where $\mathbb{S}^{\bullet}$ is an object in the bounded derived category $D^{b}(Y ; \mathfrak{K})$ and $d$ is an isomorphism

$$
d: \mathbb{S}^{\bullet} \rightarrow \mathfrak{D}\left(\mathbb{S}^{\bullet}\right)[m]
$$

in this category. (Here $\mathfrak{D}$ denotes the Borel-Moore-Verdier dualizing functor; see [Bo, §7].)

As an example, let $V$ be a component of a stratum of $Y$, let $\mathscr{Z}$ be a local system of finite-dimensional $\mathfrak{K}$-vector spaces on $V$, and let

$$
\mathscr{P}: \mathscr{Z} \otimes_{\mathfrak{K}} \mathscr{Z} \rightarrow \mathfrak{K}_{V}
$$

be a nonsingular bilinear pairing of local systems. Assume that $V$ has codimension $2 c$ and that the closure $\bar{V}$ is a "Witt space," i.e., a stratified pseudomanifold such that the middle-dimensional intersection homology with middle perversity vanishes on links of odd-codimension strata; see [S; GM2, §6]. (For the geometric applications in the present paper, it would be enough to assume $\bar{V}$ has only even-codimension strata.) Then, from [GM2], $\mathscr{P}$ induces an isomorphism

$$
\mathbb{I} \mathbb{C}_{\bar{m}}^{\bullet}(\bar{V} ; \mathscr{Z}) \cong \mathfrak{D}\left(\mathbb{I} \mathbb{C}_{\bar{m}}^{\bullet}(\bar{V} ; \mathscr{Z})\right)[m-2 c]
$$

and hence

$$
j_{*} \mathbb{I} \mathbb{C}_{\bar{m}}^{\bullet}(V ; \mathscr{Z})[c] \cong \mathfrak{D}\left(j_{*} \mathbb{I} \mathbb{C}_{\bar{m}}^{\bullet}(V ; \mathscr{Z})[c]\right)[m],
$$

$j_{*}$ the inclusion of $\bar{V}$ in $Y$. Thus $j_{*} \mathbb{I} \mathbb{C}_{\bar{m}}^{\bullet}(\bar{V} ; \mathscr{Z})[c]$ is a self-dual complex of sheaves on $Y$.

Given morphisms

$$
\mathbb{X}^{\bullet} \stackrel{u}{\longrightarrow} \mathbb{Y}^{\bullet} \stackrel{v}{\longrightarrow} \mathbb{Z}^{\bullet}
$$

in $D^{b}(Y)$ with $v \circ u$ trivial, let $\mathbb{C}_{u, v}^{\bullet}=\mathbb{C}_{u^{\prime}}^{\bullet}$ be the algebraic mapping cylinder of a lift of $u$ to $\mathbb{C}_{v}^{\bullet}[-1]$. Assuming $\operatorname{Hom}_{D^{b}}\left(\mathbb{X}^{\bullet}, \mathbb{Z}^{\bullet}\right)[-1]=0$, this will be well defined. The reader can check that $\mathbb{C}_{u, v}^{\bullet}$ is isomorphic to $\mathbb{C}_{v^{\prime}}^{\bullet}[-1]$, where $v^{\prime}$ is a factorization of $v$ through $\mathbb{C}_{u}^{\bullet}$. In particular, from the isomorphisms $\mathfrak{D} \mathbb{C}_{w}^{\bullet} \cong \mathbb{C}_{\mathfrak{D} w}^{\bullet}[-1], w=v$ or $u^{\prime}$, it follows that $\mathfrak{D} \mathbb{C}_{v, u}^{\bullet} \cong \mathbb{C}_{\mathfrak{D} u, \mathfrak{D} v}^{\bullet}$.

Now suppose that $\mathbb{Y}^{\bullet}$ is a self-dual complex of sheaves on $Y$, and that we are given an isomorphism $\mathbb{Z}^{\bullet} \cong \mathfrak{D}\left(\mathbb{X}^{\bullet}\right)[m]$ such that the following commutes.

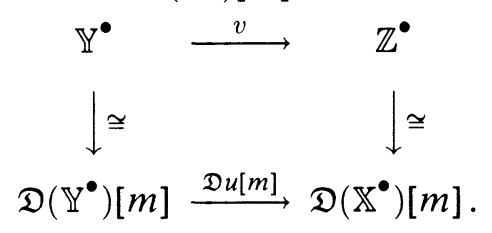


This will usually be abbreviated by writing $v=\mathfrak{D} u[m]$; with this notation we obtain an isomorphism

$$
\mathbb{C}_{v, u}^{\bullet} \cong \mathfrak{D} \mathbb{C}_{\mathfrak{D} u, \mathfrak{D} v}^{\bullet}=\mathfrak{D}\left(\mathbb{C}_{v, u}[-m]\right)=\mathfrak{D}\left(\mathbb{C}_{v, u}^{\bullet}\right)[m] .
$$

Thus $\mathbb{Y}_{i}^{\bullet}$ is also a self-dual complex of sheaves. We will say in this circumstance that $\mathbb{Y}_{1}^{\bullet}$ is obtained from $\mathbb{Y}^{\bullet}$ by an elementary cobordism. We will say that $\mathbb{Y}^{\bullet}$ is cobordant to $\hat{\mathbb{Y}}^{\bullet}$ if there is a sequence $\mathbb{Y}^{\bullet}=\mathbb{Y}_{0}^{\bullet}, \mathbb{Y}_{1}^{\bullet}, \ldots, \mathbb{Y}_{t}^{\bullet}=\widehat{\mathbb{Y}}^{\bullet}$ such that $\mathbb{Y}_{i}^{\bullet}$ is obtained from $\mathbb{Y}_{i-1}^{\bullet}$ by an element cobordism. (Exercise. Show that cobordism is an equivalence relation.)

Let $\mathbb{S}^{\bullet}$ be a self-dual sheaf over $Y$. For each component $V$ of a stratum of $Y$ of codimension $2 c(V)$, let

$$
\mathscr{Y}^{V}\left(\mathbb{S}^{\bullet}\right)=\mathbb{H}^{c(V)-m}\left(j_{V}^{!} \mathbb{S}^{\bullet}\right) .
$$

The morphism

$$
j_{V}^{!} \mathbb{S}^{\bullet} \rightarrow j_{V}^{*} \mathbb{S}^{\bullet} \stackrel{d}{\longrightarrow} j_{V}^{*} \mathfrak{D}\left(\mathbb{S}^{\bullet}\right)[m]=\mathfrak{D}\left(j_{V}^{!} \mathbb{S}^{\bullet}\right)[m]
$$

induces upon applying $\mathbb{H}^{c(V)-m}(\cdots)_{y}$ a map

$$
\mathscr{Y}^{V}\left(\mathbb{S}^{\bullet}\right)_{y} \rightarrow \operatorname{Hom}_{\mathfrak{\kappa}}\left(\mathscr{Y}^{V}\left(\mathbb{S}^{\bullet}\right)_{y}, \mathbb{H}^{-\operatorname{dim} V}\left(\mathbb{D}_{V}^{\bullet}\right)_{y}\right)
$$

of local systems. The orientation provides an isomorphism $\mathbb{D}_{V}^{\bullet} \cong \mathfrak{K}[\operatorname{dim} V]$. Therefore we have a map of local systems

$$
\mathscr{Y}^{V}\left(\mathbb{S}^{\bullet}\right) \rightarrow \operatorname{Hom}_{\mathfrak{\kappa}}\left(\mathscr{Y}^{V}\left(\mathbb{S}^{\bullet}\right), \mathfrak{K}_{V}\right)=\mathscr{Y}^{V}\left(\mathbb{S}^{\bullet}\right)^{*},
$$

and hence a nonsingular bilinear pairing

$$
\mathscr{P}_{V}\left(\mathbb{S}^{\bullet}\right): \mathscr{Z}^{V}\left(\mathbb{S}^{\bullet}\right) \otimes_{R} \mathscr{Z}^{V}\left(\mathbb{S}^{\bullet}\right) \rightarrow \mathfrak{K}_{V}
$$

on the image local system $\mathscr{Z}^{V}\left(\mathbb{S}^{\bullet}\right)$.

(2.1) Theorem. Let $\mathbb{S}^{\bullet}$ be a self-dual complex of sheaves over $Y$. Assume that $Y$ has only even-codimension strata. Then $\mathbb{S}^{\bullet}$ is cobordant to the orthogonal sum

$$
\sum_{\mathscr{V}} j_{*} \mathbb{I C}_{\bar{m}}^{\bullet}\left(\bar{V} ; \mathscr{Z}^{V}\left(\mathbb{S}^{\bullet}\right)\right)[c(V)],
$$

$\mathscr{V}$ the collection of components of strata and $\operatorname{codim} V=2 c(V)$.

The proof will be given in the next section.

Let

$$
\beta: \mathbb{B}^{\bullet} \rightarrow \mathfrak{D}\left(\mathbb{B}^{\bullet}\right)[m-1]
$$

be a morphism in $D^{b}\left(Y^{m}\right)$ satisfying

$$
\beta= \pm \mathfrak{D}(\beta)[m-1] .
$$

Then $\beta$ induces an isomorphism

$$
d_{\beta}: \mathbb{S}_{\beta} \rightarrow \mathfrak{D}\left(\mathbb{S}_{\beta}^{\bullet}\right)[m],
$$


where $\mathbb{S}_{\beta}^{\bullet}$ is defined by the triangle

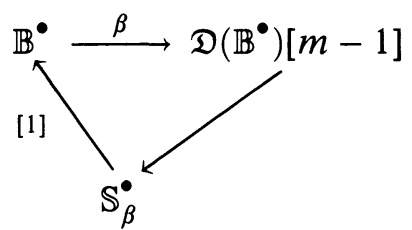

(Compare [GS].) We will say that the self-dual complex of sheaves $\left(\mathbb{S}^{\bullet}, d\right)$ is the boundary of $\beta$,

$$
\left(\mathbb{S}^{\bullet}, d\right)=\partial \beta,
$$

if there is an isomorphism in the derived category

$$
\left(\mathbb{S}^{\bullet}, d\right) \cong\left(\mathbb{S}_{\beta}^{\bullet}, d_{\beta}\right) \text {. }
$$

(2.2) Proposition. If the self-dual complex $\mathbb{S}^{\bullet}$ is cobordant to $\mathbb{S}_{1}^{\bullet}$ and if $\mathbb{S}^{\bullet}$ is a boundary, so is $\mathbb{S}_{1}^{\bullet}$.

We leave the proof as an exercise. We also note that if $\mathbb{S}^{\bullet}$ is a boundary, then it is (obviously) cobordant to the trivial complex $0^{\circ}$. Thus, it follows that $\mathbb{S}^{\bullet}$ is a boundary if and only if $\mathbb{S}^{\bullet}$ is cobordant to $0^{\bullet}$.

Suppose now that $Y^{m}$ is compact and that $m=2 n$. Let $\mathbb{S}^{\bullet}$ be a self-dual complex of sheaves on $Y$. Then $d$ induces an isomorphism on hypercohomology

$$
\mathscr{H}^{-n}\left(Y ; \mathbb{S}^{\bullet}\right) \rightarrow \mathscr{H}^{n}\left(Y ; \mathfrak{D}\left(\mathbb{S}^{\bullet}\right)\right) \cong \mathscr{H}^{-n}\left(Y ; \mathbb{S}^{\bullet}\right)^{*},
$$

and hence a nonsingular bilinear pairing

$$
\mathscr{Q}_{\mathbb{S}^{\bullet}}: \mathscr{H}^{-n}\left(Y ; \mathbb{S}^{\bullet}\right) \otimes_{\mathfrak{K}} \mathscr{H}^{-n}\left(Y ; \mathbb{S}^{\bullet}\right) \rightarrow \mathfrak{K} .
$$

If $\mathbb{S}^{\bullet}$ is a boundary, then $d= \pm \mathfrak{D}(d)[m]$, and hence $\mathscr{Q}_{\mathbb{S}^{\bullet}}$ is also symmetric or skew-symmetric.

(2.3) Proposition. Let the symmetric or skew-symmetric self-dual complexes of sheaves $\mathbb{S}_{1}^{\bullet}$ and $\mathbb{S}_{2}^{\bullet}$ on $Y^{2 n+1}$, assumed compact, be cobordant. Then $\mathscr{Q}_{\mathbb{S}_{1}^{\bullet}}$ and $\mathscr{Q}_{\mathbb{S}_{2}^{*}}$ represent the same element in the Witt group $\mathscr{W}(\mathfrak{K}, \varepsilon)$ of nonsingular symmetric $(\varepsilon=1)$ or skew-symmetric $(\varepsilon=-1)$ bilinear forms over $\mathfrak{K}$.

In particular, if $\mathbb{S}^{\bullet}$ is a boundary, then $\mathscr{Q}_{\mathbb{S}^{\bullet}}$ represents zero in the Witt group. Proof (compare [GM1, §6]). We prove the last statement first. Let $\left(\mathbb{S}^{\bullet}, d\right)=$ $\partial \beta$

$$
\beta: \mathbb{B}^{\bullet} \rightarrow \mathfrak{D}\left(\mathbb{B}^{\bullet}\right)[m-1]=\mathbb{A}^{\bullet} .
$$

Then the exact sequence

$$
\begin{gathered}
\mathscr{H}^{-n}\left(Y ; \mathbb{B}^{\bullet}\right) \rightarrow \mathscr{H}^{-n}\left(Y ; \mathbb{A}^{\bullet}\right) \rightarrow \mathscr{H}^{-n}\left(Y ; \mathbb{S}^{\bullet}\right) \\
\rightarrow \mathscr{H}^{-n+1}\left(Y ; \mathbb{B}^{\bullet}\right) \rightarrow \mathscr{H}^{-n+1}\left(Y ; \mathbb{A}^{\bullet}\right)
\end{gathered}
$$

is dually paired to itself, with the pairing on the middle group being $\mathscr{Q}_{\mathbb{S}} \bullet$. Hence the image of

$$
\mathscr{H}^{-n}\left(Y ; \mathbb{A}^{\bullet}\right) \rightarrow \mathscr{H}^{-n}\left(Y ; \mathbb{S}^{\bullet}\right)
$$


is a self-annihilating subspace of dimension one-half that of $\mathscr{H}^{-n}\left(Y ; \mathbb{S}^{\bullet}\right)$, i.e., $\mathscr{Q}_{\mathbb{S}^{\bullet}}$ represents zero in the Witt group.

In analogy with a familiar fact for forms, it is not difficult to see that $\left(\mathbb{S}_{2}^{\bullet}, d_{2}\right) \oplus\left(\mathbb{S}_{2}^{\bullet},-d_{2}\right)$ is isomorphic to $\mathbb{S}_{2}^{\bullet} \oplus \mathfrak{D}\left(\mathbb{S}_{2}^{\bullet}\right)[m]$ with the obvious duality map; i.e., $\mathbb{S}_{2}^{\bullet} \oplus-\mathbb{S}_{2}^{\bullet}$ is the boundary of

$$
\mathbb{S}_{2}^{\bullet} \stackrel{0}{\longrightarrow} \mathfrak{D}\left(\mathbb{S}_{2}^{\bullet}\right)[m-1]
$$

Clearly, $\mathbb{S}_{2}^{\bullet} \oplus-\mathbb{S}_{2}^{\bullet}$ is cobordant to $\mathbb{S}_{1}^{\bullet} \oplus-\mathbb{S}_{2}^{\bullet}$. Hence it is also a boundary, by (2.2). Hence

$$
\mathscr{Q}_{\mathbb{S}_{1}^{\bullet} \oplus-\mathbb{S}_{2}^{\bullet}}=\mathscr{Q}_{\mathbb{S}_{1}^{\bullet}} \oplus-\mathscr{Q}_{\mathbb{S}_{2}^{\bullet}}
$$

represents zero in the Witt group.

Now suppose $\mathbb{S}^{\bullet}$ is a self-dual complex of sheaves, as in (2.1), and let $V$ be the interior of a stratum of codimension $2 c$ in $Y^{2 n}$ with $\bar{V}$ a Witt space. Then $j_{*} \mathbb{I} \mathbb{C}_{\bar{m}}^{\bullet}\left(\bar{V} ; \mathscr{Z}^{V}\left(\mathbb{S}^{\bullet}\right)\right)[c], j$ the inclusion of $\bar{V}$, is a self-dual complex of sheaves. Clearly,

$$
\mathscr{H}^{-n}\left(Y ; j_{*} \mathbb{I}_{\bar{m}}^{\bullet}\left(\bar{V} ; \mathscr{Z}^{V}\left(\mathbb{S}^{\bullet}\right)[c]\right)\right)=I H_{n-c}^{\bar{m}}\left(\bar{V} ; \mathscr{Z}^{V}\left(\mathbb{S}^{\bullet}\right)\right)
$$

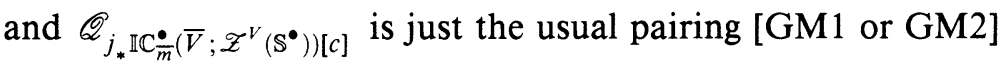

$$
\mathscr{P}_{V}\left(\mathbb{S}^{\bullet}\right)_{*}: I H_{n-c}^{\bar{m}}\left(\bar{V} ; \mathscr{Z}^{V}\left(\mathbb{S}^{\bullet}\right)\right) \otimes_{\mathfrak{K}} I H_{n-c}^{\bar{m}}\left(V ; \mathscr{Z}^{V}\left(\mathbb{S}^{\bullet}\right)\right) \rightarrow \mathfrak{K}
$$

induced by $\mathscr{P}_{V}\left(\mathbb{S}^{\bullet}\right)$ and the orientation of $V$. Let $\left[\mathscr{P}_{V}\left(\mathbb{S}^{\bullet}\right)_{*}\right]$ be the element in the appropriate Witt group represented by this pairing. Then, combining (2.1), (2.2), and (2.3), we obtain

(2.4) Corollary. Let $Y^{m}$ and $\mathbb{S}^{\bullet}$ be as in (2.1), and assume in addition that $Y$ is compact and that $m=2 n$. Then, in the Witt group $\mathscr{W}(\mathfrak{K}, \varepsilon)$,

$$
\sum_{\mathscr{V}}\left[\mathscr{P}_{V}\left(\mathbb{S}^{\bullet}\right)_{*}\right]=\left[\mathscr{Q}_{\mathbb{S}^{\bullet}}\right]
$$

\section{Proof of (2.1)}

(3.1) Lemma. Assume, in addition to the hypotheses of (2.1), that $\mathbb{S}^{\bullet}$ is perverse with respect to the middle perversity, i.e., that for $V$ a stratum of codimension $2 c$ the restriction

$$
\mathbb{H}^{i} \mathbb{S}^{\bullet} \mid V=0
$$

for $i>c-m$. Then the conclusion of (2.1) holds for $\mathbb{S}^{\bullet}$. 
(Note. By definition, a perverse sheaf is an object in $D^{b}(Y ; \mathfrak{K})$ that satisfies the above vanishing and the condition

$$
\mathbb{H}^{i}\left(j_{V}^{!} \mathbb{S}^{\bullet}\right)=0
$$

for $i<c-m, V$ a component of a stratum of $Y$, and $j_{V}$ the inclusion of $V$ in $Y$. For a self-dual complex of sheaves, these two vanishing conditions are equivalent to one another.)

Proof of (3.1). Let $V$ be a component of the stratum of maximal codimension $2 c$ such that the local system

$$
\mathscr{Y}^{V}=\mathscr{Y}^{V}\left(\mathbb{S}^{\bullet}\right)=\mathbb{H}^{c-m}\left(j_{V}^{!} \mathbb{S}^{\bullet}\right)
$$

is not trivial. Let $j=j_{\bar{V}}$ be the inclusion of the closure $\bar{V}$ in $Y$. Then it is

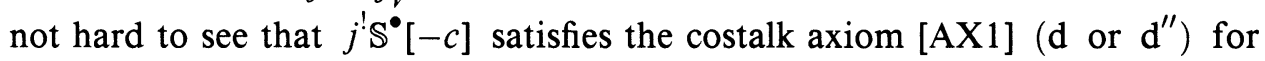
the middle perversity $\bar{m}$. Hence, by (1.3), there exists a unique morphism

$$
\lambda_{0}: \mathbb{I C}_{\bar{m}}^{\bullet}\left(\bar{V} ; \mathscr{Y}^{V}\right)[c] \rightarrow j ! \mathbb{S}^{\bullet}
$$

that induces the identity on stalks over $V$, in dimension $c-m$.

Let $\bar{m}_{j} !$ be the functor on the category of $\bar{m}$-perverse sheaves over $\mathfrak{K}$, associated to $j^{\prime}$; in the notation of [BBD, 2.1.7],

$$
{ }^{\bar{m}} j ! \mathbb{S}^{\bullet}={ }^{\bar{m}} \tau_{\leq 0} j^{\prime} \mathbb{S}^{\bullet}=\tau_{\leq \bar{m}} j ! \mathbb{S}^{\bullet}=H^{\bar{m}}\left(j ! \mathbb{S}^{\bullet}\right) .
$$

Thus, $j_{*}^{\bar{m}} j^{!} \mathbb{S}^{\bullet}$ will be an $\bar{m}$-perverse sheaf over $Y$, or equivalently, ${ }^{\bar{m}} j ! \mathbb{S}^{\bullet}[-c]$ will be an $m$-perverse sheaf over $\bar{V}$. (Recall we are using the indexing conventions of [GM2].)

Consider the object $\mathbb{E}^{\bullet}$ defined by the distinguished triangle

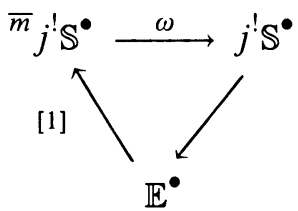

Then, by (2.1.4) and (1.3.3) of [BBD], for every object in ${ }^{\bar{m}} D^{b, \leq 0}(Y ; \mathfrak{K})$, and in particular for every $\bar{m}$-perverse sheaf $\mathbb{P}^{\bullet}$ over $Y$

$$
\operatorname{Hom}_{D^{b}(Y)}\left(\mathbb{P}^{\bullet}, j_{*} \mathbb{E}^{\bullet}\right)=\operatorname{Hom}_{D^{b}(Y)}\left(\mathbb{P}^{\bullet}, j_{*} \mathbb{E}^{\bullet}[-1]\right)=0 .
$$

Since $j_{*} \mathbb{I} \mathbb{C}_{\bar{m}}^{\bullet}\left(\bar{V} ; \mathscr{Y}^{V}\right)[c]$ is $m$-perverse, it follows that there is a unique morphism

satisfying

$$
\lambda: \mathbb{I} \mathbb{C}_{\bar{m}}^{\bullet}\left(\bar{V} ; \mathscr{Y}^{V}\right)[c] \rightarrow^{\bar{m}} j ! \mathbb{S}^{\bullet}
$$

$$
\lambda_{0}=\omega \circ \lambda .
$$

Using the duality map, we have an isomorphism

$$
\begin{aligned}
\mathbb{H}^{c-m}\left(j_{V}^{*} \mathbb{S}^{\bullet}\right) & \cong \mathbb{H}^{c-m}\left(j_{V}^{*} \mathfrak{D}\left(\mathbb{S}^{*}\right)[m]\right)=\mathbb{H}^{c}\left(\mathfrak{D}\left(j_{V}^{!} \mathbb{S}^{\bullet}\right)\right) \\
& =\mathbb{H}^{c}(\mathfrak{D}(\mathscr{Y}[m-2 c]))=\left(\mathscr{Y}^{V}\right)^{*},
\end{aligned}
$$


where

$$
\left(\mathscr{Y}^{V}\right)_{y}^{*}=\operatorname{Hom}_{\mathfrak{K}}\left(\mathscr{Y}_{y}^{V} ; \mathfrak{K}\right) .
$$

We will make the identification

$$
\mathbb{H}^{c-m}\left(j_{V}^{*} \mathbb{S}^{\bullet}\right)=\left(\mathscr{Y}^{V}\right)^{*} .
$$

By the duality of $\mathbb{S}^{\bullet}, V$ is also a component of the stratum of maximal codimension such that

$$
\mathbb{H}^{c-m}\left(j_{V}^{*} \mathbb{S}^{\bullet}\right) \neq 0 .
$$

Hence $j^{*} \mathbb{S}^{\bullet}[-c]$ satisfies the stalk axiom $[\mathrm{AX} 1](\mathrm{c})$ for the middle perversity. Hence there exists a unique morphism in $D^{b}(\bar{V} ; \mathfrak{K})$

$$
\mu_{0}: j^{*} \mathbb{S}^{\bullet} \rightarrow \mathbb{I} \mathbb{C}_{\bar{m}}^{\bullet}\left(\bar{V} ;\left(\mathscr{Y}^{V}\right)^{*}\right)[c]
$$

inducing the identity on stalks at points of $V$, in dimension $c-m$.

As for $j$, we have the $\bar{m}$-perverse sheaf

$$
{ }^{\bar{m}} j^{*} \mathbb{S}^{\bullet}={ }^{\bar{m}} \tau_{\geq 0} j^{*} \mathbb{S}^{\bullet}=\tau_{\geq \bar{m}} j^{*} \mathbb{S}^{\bullet}=H^{\bar{m}}\left(j^{*} \mathbb{S}^{\bullet}\right),
$$

and an argument similar to the above shows that there is a unique morphism

$$
\mu:{ }^{\bar{m}} j^{*} \mathbb{S}^{\bullet} \rightarrow \mathbb{I} \mathbb{C}_{\bar{m}}^{\bullet}\left(\bar{V} ;\left(\mathscr{Y}^{V}\right)^{*}\right)[c]
$$

such that

$$
\mu_{0}=\mu \circ \psi
$$

where

$$
\psi:{ }^{\bar{m}} j^{*} \mathbb{S}^{\bullet} \rightarrow j^{*} \mathbb{S}^{\bullet}
$$

is the canonical map.

Consider the diagram

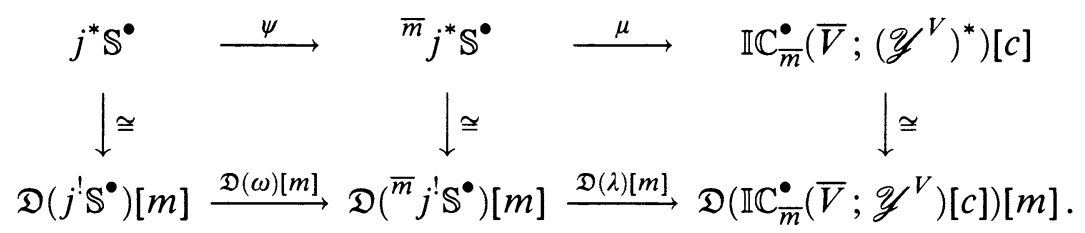

The left and middle vertical arrows are induced by $d$ and the right is just the duality of the intersection complex. (Strictly speaking, the target of the middle isomorphism should be $\mathfrak{D}\left(\tau_{\leq \bar{n}} j^{!} \mathbb{S}^{\bullet}\right)[m]$. Since $\bar{V}$ has no strata of odd codimension, ${ }^{\bar{m}} j ! \mathbb{S}^{\bullet}=\tau_{<\bar{m}} j ! \mathbb{S}^{\bullet}=\tau_{<\bar{n}} j ! \mathbb{S}^{\bullet}$ in this case.) The left square commutes for general reasons [BBD] and the rectangle commutes by the uniqueness of $\mu_{0}$. Hence, by the uniqueness of $\mu$ factoring $\mu_{0}$ through $\omega$, the right square also commutes. We will write this as

$$
\mu=\mathfrak{D}(\lambda)[m] \text {. }
$$

Let $\mathfrak{X}^{V}$ be the kernel of the map $\mathscr{Y}^{V} \rightarrow\left(\mathscr{Y}^{V}\right)^{*}$ obtained upon application of $\mathbb{H}^{c-m}(\cdots) \mid V$ to the canonical morphism

$$
j ! \mathbb{S}^{\bullet} \rightarrow j^{*} \mathbb{S}^{\bullet}
$$


then there is an exact sequence

$$
0 \rightarrow \mathfrak{X}^{V} \rightarrow \mathscr{Y}^{V} \rightarrow\left(\mathscr{Y}^{V}\right)^{*} \rightarrow\left(\mathfrak{X}^{V}\right) \rightarrow 0
$$

Let

$$
\mathscr{Z}^{V}=\mathscr{Y}^{V} / \mathfrak{X}^{V}
$$

Then the map $\mathscr{Y}^{V} \rightarrow\left(\mathscr{Y}^{V}\right)^{*}$ induces an isomorphism

$$
\varphi_{V}: \mathscr{Z}^{V} \rightarrow\left(\mathscr{Z}^{V}\right)^{*}=\operatorname{ker}\left(\left(\mathscr{Y}^{V}\right)^{*} \rightarrow\left(\mathscr{Y}^{V}\right)^{*}\right),
$$

that is the adjoint of a nonsingular bilinear pairing

$$
\mathscr{P}_{V}: \mathscr{Z}^{V} \otimes_{\mathfrak{K}} \mathscr{Z}^{V} \rightarrow \mathfrak{K} .
$$

By (1.3), the composite morphism

$$
{ }^{\bar{m}} !^{\bullet} \mathbb{S}^{\bullet}[-c] \stackrel{\lambda[-c]}{\longrightarrow} \mathbb{I} \mathbb{C}_{\bar{m}}^{\bullet}\left(\bar{V} ;\left(\mathscr{Y}^{V}\right)^{*}\right) \rightarrow \mathbb{I} \mathbb{C}_{\bar{m}}^{\bullet}\left(\bar{V} ;\left(\mathfrak{X}^{V}\right)^{*}\right)
$$

is trivial. Since the exact sequence

$$
0 \rightarrow \mathscr{Z}^{V} \rightarrow\left(\mathscr{Y}^{V}\right)^{*} \rightarrow\left(\mathfrak{X}^{V}\right)^{*} \rightarrow 0
$$

splits (not naturally) over the field $\mathfrak{K}$, we also have the distinguished triangle

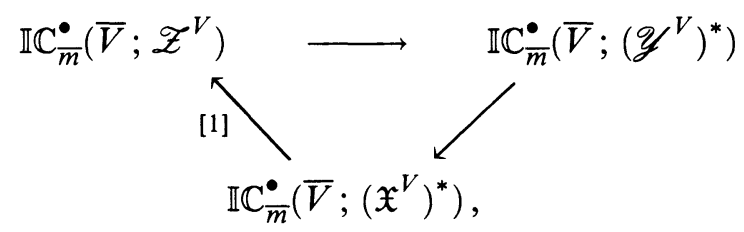

in which the map raising degrees induces zero on stalks and costalks and the other maps are the obvious ones. Hence there is a morphism

$$
\lambda_{1}:{ }^{\bar{m}} j ! \mathbb{S}^{\bullet} \rightarrow \mathbb{I} \mathbb{C}_{\bar{m}}^{\bullet}\left(\bar{V} ; \mathscr{Z}^{V}\right)[c],
$$

unique by (1.3), that induces the quotient projection $\mathscr{Y}^{V} \rightarrow \mathscr{Z}^{V}$ on $\mathbb{H}^{c-m}$ restricted to $V$.

Similarly, there exists a unique morphism

$$
\mu_{1}: \mathbb{I} \mathbb{C}_{\bar{m}}^{\bullet}\left(\bar{V} ;\left(\mathscr{Z}^{V}\right)^{*}\right)[c] \rightarrow{ }^{\bar{m}} j^{*} \mathbb{S}^{\bullet}
$$

inducing the inclusion of $\left(\mathscr{Z}^{V}\right)^{*}$ in $\left(\mathscr{Y}^{V}\right)^{*}$. By uniqueness, these correspond under duality, as for $\lambda$ and $\mu$; i.e.,

$$
\mu_{1}=\mathfrak{D}\left(\lambda_{1}\right)[m] \text {. }
$$

Hence if $\mathbb{X}_{1}^{\bullet}$ and $\mathbb{Z}_{1}^{\bullet}$ and $u_{1}$ and $v_{1}$ are defined by distinguished triangles

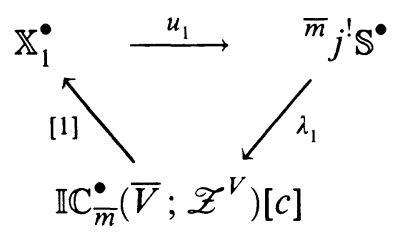


and

then there is an induced isomorphism

$$
\mathbb{I C}_{\bar{m}}^{\bullet}\left(\bar{V} ;\left(\mathscr{Z}^{V}\right)^{*}\right)[c] \stackrel{\mu_{1}}{\longrightarrow} \overbrace{\mathbb{Z}_{1}^{\bullet}}^{\bar{m}} j^{*} \mathbb{S}^{\bullet}
$$

$$
\mathbb{Z}_{1}^{\bullet} \cong \mathfrak{D}\left(\mathbb{X}_{1}^{\bullet}\right)[m]
$$

and

$$
v_{1}=\mathfrak{D}\left(u_{1}\right)[m]
$$

Note that $\lambda_{1} \circ \lambda[-c]$ is the unique morphism of intersection complexes induced by the surjection $\mathscr{Y}^{V} \rightarrow \mathscr{Z}^{V}$, which splits over the field $\mathfrak{K}$. Hence this composite, and therefore $\lambda_{1}$ as well, induces surjections on stalks and costalks. Hence, from the first triangle above, $u_{1}$ induces injections on stalks and costalks. Similarly, or by duality, $v_{1}$ induces surjections. In particular, $j_{*} \mathbb{X}_{1}^{\bullet}=\mathbb{X}^{\bullet}$ and $j_{*} \mathbb{Z}_{1}^{\bullet}=\mathbb{Z}^{\bullet}$ are $\bar{m}$-perverse sheaves on $Y, \mathbb{X}_{1}^{\bullet}[-c]$ satisfies the costalk axiom of [AX1] (for $\bar{m}$ ), and $\mathbb{Z}^{\bullet}[-c]$ the stalk axiom.

By the injectivity statement in (1.3), the square in the diagram

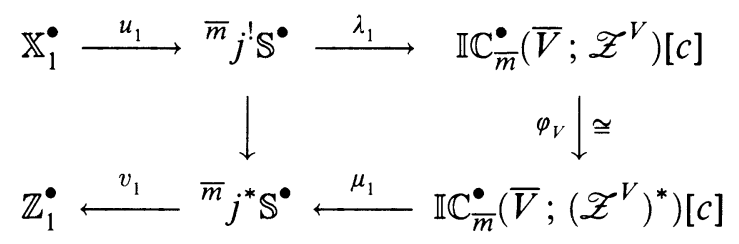

commutes. Hence the composite

$$
\mathbb{X}_{1}^{\bullet} \stackrel{u_{1}}{\longrightarrow}{ }^{\bar{m}} j^{!} \mathbb{S}^{\bullet} \longrightarrow{ }^{\bar{m}} j^{*} \mathbb{S}^{\bullet} \stackrel{v_{1}}{\longrightarrow} \mathbb{Z}_{1}^{\bullet}
$$

is trivial.

Let $u$ and $v$ be the composites

$$
\mathbb{X}^{\bullet}=j_{*} \mathbb{A}_{1}^{\bullet} \stackrel{j_{*} u_{1}}{\longrightarrow} j_{*}^{\bar{m}} j ! \mathbb{S}^{\bullet} \longrightarrow \mathbb{S}^{\bullet}
$$

and

$$
\mathbb{S}^{\bullet} \longrightarrow j_{*}^{\bar{m}} j^{*} \mathbb{S}^{\bullet} \stackrel{j_{*} v_{1}}{\longrightarrow} j_{*} \mathbb{Z}_{1}^{\bullet}=\mathbb{Z} .
$$

Then from the preceding, we obtain an isomorphism

$$
\mathbb{Z}^{\bullet} \cong \mathfrak{D}\left(\mathbb{X}^{\bullet}\right)[m]
$$

and we have that

$$
v=\mathfrak{D}(u)[m],
$$

and that $v \circ u=0$. Hence we can construct an elementary cobordism from $\mathbb{S}^{\bullet}$ to the self-dual complex $\mathbb{S}_{0}^{\bullet}=\mathbb{C}_{u, v}^{\bullet}$.

Clearly $\mathbb{S}_{0}^{\bullet}\left|(Y-\bar{V}) \cong \mathbb{S}^{\bullet}\right|(Y-\bar{V})$. It also follows readily that

$$
{ }^{\bar{m}}{ }_{j} \mathbb{S}_{0}^{\bullet}=\mathbb{I} \mathbb{C}_{\bar{m}}^{\bullet}\left(\bar{V} ; \mathscr{Z}^{V}\right) \oplus \mathbb{X}_{1}^{\bullet}[-1],
$$


that

$$
\bar{m}^{*} \mathbb{S}_{0}^{\bullet}=\mathbb{I} \mathbb{C}_{\bar{m}}^{\bullet}\left(\bar{V} ;\left(\mathscr{Z}^{V}\right)^{*}\right) \oplus \mathbb{Z}_{1}^{\bullet}[1],
$$

and, with the aid of (1.3), that the canonical morphism between these is the sum of the isomorphism induced by

$$
\varphi_{V}: \mathscr{Z}^{V} \rightarrow\left(\mathscr{Z}^{V}\right)^{*}
$$

and the zero map on $\mathbb{X}_{1}^{\bullet}[-1]$. This implies that $\mathbb{S}_{0}^{\bullet}$ has an orthogonal decomposition

$$
\mathbb{S}_{0}^{\bullet}=j_{*} \mathbb{I} \mathbb{C}_{\bar{m}}^{\bullet}\left(\bar{V} ; \mathscr{Z}^{V}\right)[c] \oplus \widehat{\mathbb{S}}^{\bullet},
$$

where $\widehat{\mathbb{S}}^{\bullet}$ is a perverse self-dual sheaf on $Y$ satisfying

$$
\mathbb{H}^{c(W)-m}\left(j_{W}^{!} \widehat{\mathbb{S}}^{\bullet}\right)=0,
$$

for $W$ a stratum of $Y$ of codimension $2 c(W)>2 c(V)$ and for $W=V$.

Hence by induction we see that $\mathbb{S}^{\bullet}$ is cobordant to a sum of intersection complexes, as in the statement of the result, and a perverse self-dual sheaf $\mathbb{S}_{1}^{\bullet}$ with $\mathbb{H}^{c(V)-m}\left(j_{V}^{!} \mathbb{S}_{1}^{\bullet}\right)$ trivial for all strata $V$ of $Y \cdot$. By duality, $\mathbb{H}^{c(V)-m}\left(j_{V}^{*} \mathbb{S}_{1}^{\bullet}\right)$ is also trivial for all strata $V$ of $Y$. Applying this to the singular strata, we see that $\mathbb{S}_{1}^{\bullet}$ satisfies the stalk and costalk axioms $[A X 1](c, d)$ for the intersection complex with the middle perversity. For $V=Y-Y_{m-2}, j_{V}^{!} \mathbb{S}_{1}^{\bullet}=j_{V}^{*} \mathbb{S}_{1}$; hence $\mathbb{S}_{1}^{\bullet} \mid\left(Y-Y_{m-2}\right) \cong 0$. It follows by [GM2, 3 ; Bo], or (1.3) that $\mathbb{S}_{1}^{\bullet} \cong 0$.

We note that in the proof, when $\mathscr{Y}^{V} \rightarrow\left(\mathscr{Y}^{V}\right)^{*}$ is an isomorphism, i.e., when $\mathscr{Y}^{V}=\mathscr{Z}^{V}$, no cobordism is required before splitting off an intersection complex. This is always the case for the top stratum in the support of $\mathbb{S}^{\bullet}$. If this holds for all $V \in \mathscr{V}$, we say that $\mathbb{S}^{\bullet}$ is locally nonsingular. Thus

(3.2) Theorem. Let $\mathbb{S}^{\bullet}$ be a perverse locally nonsingular self-dual sheaf over $Y$. Then in $D^{b}(Y ; \mathfrak{K})$ there is a (canonical) isomorphism

$$
\mathbb{S}^{\bullet} \cong \sum_{\mathscr{V}} j_{*} \mathbb{I} \mathbb{C}_{\bar{m}}^{\bullet}\left(\bar{V} ; \mathscr{Z}^{V}\left(\mathbb{S}^{\bullet}\right)\right)[c(V)],
$$

$\mathscr{V}$ the collection of components of strata and $\operatorname{codim} V=2 c(V)$.

The next lemma will complete the proof of (2.1).

(3.3) Lemma. Let $\mathbb{S}^{\bullet}$ be a self-dual complex of sheaves on the stratified space $Y$ with even-codimension strata. Then $\mathbb{S}^{\bullet}$ is cobordant to $\mathbb{S}_{1}^{\bullet}$, a perverse self-dual complex of sheaves, with $\left(\mathscr{Z}^{V}\left(\mathbb{S}^{\bullet}\right), \mathscr{P}_{V}\left(\mathbb{S}^{\bullet}\right)\right)$ and $\left(\mathscr{Z}^{V}\left(\mathbb{S}_{1}^{\bullet}\right), \mathscr{P}_{V}\left(\mathbb{S}_{1}^{\bullet}\right)\right)$ isomorphic.

Proof. In fact, let

$$
\left(\mathbb{S}_{1}^{\bullet}, d_{1}\right)=\left(H^{\bar{m}}\left(\mathbb{S}^{\bullet}\right), H^{\bar{m}}(d)\right),
$$

where

$$
H^{\bar{m}}=\tau_{\geq \bar{m}} \tau_{\leq \bar{m}}=\tau_{\leq \bar{m}} \tau_{\geq \bar{m}}
$$


is the functor of [BBD, 2.1.7] from $D^{b}(Y ; \mathfrak{K})$ to the category of $m$-perverse sheaves on $Y$ over $\mathfrak{K}$. There are triangles

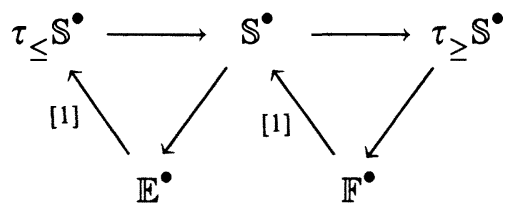

with $\mathbb{E}^{\bullet} \in{ }^{\bar{m}} D^{b, \geq 1}(Y ; \mathfrak{K})$ [BBD, 2.1 and 1.3]. Further, with [BBD, 2.1.16], the diagram

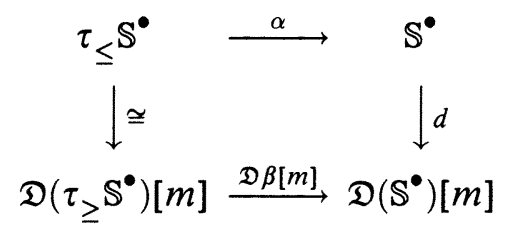

commutes. Hence there is an induced isomorphism

$$
\mathbb{E}^{\bullet} \cong \mathfrak{D}\left(\mathbb{F}^{\bullet}[-1]\right)[m],
$$

and with respect to this isomorphism and $d$, we have

$$
v=\mathfrak{D}(u)[m],
$$

where

$$
u=w[-1]: \mathbb{F}^{\bullet}[-1] \rightarrow \mathbb{S}^{\bullet} .
$$

In particular, $\mathbb{F}^{\bullet}[-1] \in \in^{\bar{m}} D^{b, \leq-1}(Y ; \mathfrak{K})$; hence (see [BBD, 1.3.1] and compare $(1.3))$

$$
\operatorname{Hom}_{D^{b}(Y)}\left(\mathbb{F}^{\bullet}[-1], \mathbb{E}^{\bullet}\right)=\operatorname{Hom}_{D^{b}(Y)}\left(\mathbb{F}^{\bullet}[-1], \mathbb{E}^{\bullet}[-1]\right)=0 .
$$

It now follows that

$$
H^{\bar{m}} \mathbb{S}^{\bullet}=\tau_{\geq \bar{m}} \tau_{\leq \bar{m}} \mathbb{S}^{\bullet}=\tau_{\leq \bar{m}} \tau_{\geq \bar{m}} \mathbb{S}^{\bullet}=\mathbb{C}_{u, v}^{\bullet},
$$

i.e., $\mathbb{S}^{\bullet}$ is cobordant to $\mathbb{S}_{1}^{\bullet}$.

We assert that for any object $\mathbb{A}^{\bullet}$ of $D^{b}(Y ; \mathfrak{K})$, the induced maps

$$
\mathbb{H}^{c-m}\left(j_{V}^{*} \tau_{\leq \bar{m}} \mathbb{A}^{\bullet}\right) \rightarrow \mathbb{H}^{c-m}\left(j_{V}^{*} \mathbb{A}^{\bullet}\right) \rightarrow \mathbb{H}^{c-m}\left(j_{V}^{*} \tau_{\geq \bar{m}} \mathbb{A}^{\bullet}\right)
$$

and

$$
\mathbb{H}^{c-m}\left(j_{V} \tau_{\leq \bar{m}} \mathbb{A}^{\bullet}\right) \rightarrow \mathbb{H}^{c-m}\left(j_{V}^{!} \mathbb{A}^{\bullet}\right) \rightarrow \mathbb{H}^{c-m}\left(j_{V} \tau_{\geq \bar{m}} \mathbb{A}^{\bullet}\right)
$$

are isomorphisms, $V$ a component of a stratum of $Y$ of codimension $2 c$; the final statement of the lemma then follows readily.

The assertion can be seen from the definition of the truncation functors, "par recollement," in [BBD]. Alternatively, consider, for example, the distinguished triangle

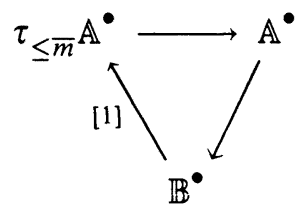


with $\mathbb{B}^{\bullet} \in{ }^{\bar{m}} D^{b, \geq 1}(Y ; \mathfrak{K})$. By definition [BBD, 2.1.2],

$$
\mathbb{H}^{i}\left(j_{V}^{!} \mathbb{B}^{\bullet}\right)=0
$$

for $i \leq c-m$. Hence

$$
\mathbb{H}^{c-m}\left(j_{V}^{!} \tau_{\leq \bar{m}} \mathbb{A}^{\bullet}\right) \rightarrow \mathbb{H}^{c-m}\left(j_{V}^{!} \mathbb{A}^{\bullet}\right)
$$

is an isomorphism.

Let $\mathscr{O}$ be a local system of $\mathfrak{K}$-modules on $V$, and let $\mathbb{I} \mathbb{C}_{\bar{m}}^{\bullet}(\mathscr{O})$ be the corresponding intersection complex on $\bar{V}$. Then, for $i \geq c, j_{*} \mathbb{I} \mathbb{C}_{\bar{m}}^{\bullet}(\mathscr{O})[i] \in{ }^{\bar{m}} D^{b, \leq 0}$ and hence

$$
\operatorname{Hom}_{D^{b}}\left(j_{*} \mathbb{I} \mathbb{C}_{\bar{m}}^{\bullet}(\mathscr{O})[i], \mathbb{B}^{\bullet}\right)=0 .
$$

An application of (1.3) yields

$$
\operatorname{Hom}_{D^{b}}\left(j_{*} \mathbb{I}_{\bar{m}}^{\bullet}(\mathscr{O})[i], \mathbb{B}^{\bullet}\right)=\operatorname{Hom}_{\mathfrak{K}}\left(\mathscr{O}, \mathbb{H}^{2 c-m-i}\left(j_{V}^{*} \mathbb{B}^{\bullet}\right)\right)
$$

Hence

$$
\operatorname{Hom}_{\mathfrak{K}}\left(\mathscr{O}, \mathbb{H}^{k}\left(j_{V^{*}}^{*} \mathbb{B}^{\bullet}\right)\right)=0
$$

for $k \leq c-m$. Taking $\mathscr{O}=\mathbb{H}^{k}\left(j_{V}^{*} \mathbb{B}^{\bullet}\right)$, we see that $\mathbb{H}^{k}\left(j_{V}^{*} \mathbb{B}^{\bullet}\right)=0$ for $k \leq c-m$ also. Hence from the above triangle,

$$
\mathbb{H}^{c-m}\left(j_{V}^{*} \tau_{\leq \bar{m}} \mathbb{A}^{\bullet}\right) \rightarrow \mathbb{H}^{c-m}\left(j_{V}^{*} \mathbb{A}^{\bullet}\right)
$$

is also an isomorphism. That the remaining maps are isomorphisms follows by similar arguments or by duality.

\section{StRatified MAPS}

Let $X$ and $Y$ be Whitney stratified subsets of smooth manifolds $M$ and $N$, respectively. Then a stratified map $f: X \rightarrow Y$ is by definition a proper map that is the restriction of a smooth map from $M$ to $N$ and that has the property that for each stratum $V$ of $Y$, the inverse image $f^{-1}(V)$ is a union of strata of $X$, each of which is mapped submersively onto $V$. (See [GM3].)

(4.1) Proposition. Let $f: X^{p} \rightarrow Y^{m}$ be a stratified map of compact Whitney stratified pseudomanifolds. Let $\mathbb{S}^{\bullet}$ be a self-dual complex of sheaves on $X$. Assume that $p-m$ is even and let $t=\frac{1}{2}(p-m)$. Then $R f_{*} \mathbb{S}^{\bullet}[-t]$ is a self-dual complex of sheaves on $Y$.

Proof. By Thom's first isotopy lemma and, e.g., [GM2, 1.9], $f_{*} \mathbb{S}^{\bullet}[-t] \in D^{b}(Y)$. Further,

$$
\mathfrak{D}\left(f_{*} \mathbb{S}^{\bullet}[-t]\right)[m] \cong f_{!} \mathfrak{D}\left(\mathbb{S}^{\bullet}\right)[t+m] \cong f_{!} \mathbb{S}^{\bullet}[t+m-p] \cong f_{*} \mathbb{S}^{\bullet}[-t]
$$

(4.2) Theorem. Let $f: X^{p} \rightarrow Y^{n}$ be a stratified map of oriented Whitney stratified spaces. Assume $X$ is compact, $Y$ has only even-codimension strata, and $t=\frac{1}{2}(p-n)$ is an integer. Let $\mathbb{S}^{\bullet}$ be a self-dual complex of sheaves on $X$. Then $f_{*} \mathbb{S}^{\bullet}[-t]$ is cobordant to

$$
\sum_{\mathscr{V}} j_{*}\left(\mathbb{I} \mathbb{C}_{\bar{m}}^{\bullet}\left(\bar{V} ; \mathscr{Z}^{V}\left(f_{*} \mathbb{S}^{\bullet}[-t]\right)\right)\right)[c(V)]
$$


Proof. Immediate from (4.1) and (2.1).

In this section we give some descriptions of the "fibres" $\mathscr{Z}^{V}\left(f_{*} \mathbb{S}^{\bullet}[-t]\right)$ and the pairings $\mathscr{P}_{V}\left(f_{*} \mathbb{S}^{\circ}[-t]\right)$ and a corresponding reformulation of (4.2). For $y \in V$, let

$$
F_{y}=f^{-1}(y) \stackrel{\gamma_{y}}{\longrightarrow} f^{-1}(V) \stackrel{\delta_{y}}{\longrightarrow} X
$$

be inclusion maps. Note that, by Thom's first isotopy lemma, $\gamma_{y}$ is normally nonsingular with trivialized normal bundle. This yields an isomorphism

$$
\gamma_{y}^{*} \delta_{y}^{*} \mathbb{S}^{\bullet} \cong \gamma_{y}^{!} \delta_{y}^{*} \mathbb{S}^{\bullet}[m-2 c] \text {, }
$$

$V$ of codimension $2 c$ in $Y$. Hence

$$
\begin{aligned}
\mathscr{H}^{c-m-t}\left(F_{y} ; \gamma_{y}^{*} \delta_{y}^{*} \mathbb{S}^{\bullet}\right) & \cong \mathscr{H}^{c+t}\left(F_{y} ; \gamma_{y}^{*} \delta_{y}^{*} \mathfrak{D} \mathbb{S}^{\bullet}\right) \\
& \cong \mathscr{H}^{c+t}\left(F_{y} ; \mathfrak{D}\left(\gamma_{y}^{\prime} \delta_{y}^{!} \mathbb{S}^{\bullet}\right)\right) \\
& \cong \operatorname{Hom}_{\mathfrak{K}}\left(\mathscr{H}^{-c-t}\left(F_{y} ; \gamma_{y} \delta_{y} \mathbb{S}^{\bullet}\right), \mathfrak{K}\right) \\
& \cong \operatorname{Hom}_{\mathfrak{K}}\left(\mathscr{H}^{c-m-t}\left(F_{y} ; \gamma_{y}^{*} \delta_{y}^{!} \mathbb{S}^{\bullet}\right), \mathfrak{K}\right),
\end{aligned}
$$

where the first isomorphism is induced by the duality of $\mathbb{S}^{\bullet}$. It is a standard fact that

$$
\mathscr{H}^{c-m-t}\left(F_{y} ; \gamma_{y}^{*} \delta_{y}^{*} \mathbb{S}^{\bullet}\right)=\mathbb{H}^{c-m}\left(j_{V}^{*} f_{*} \mathbb{S}^{\bullet}[-t]\right)_{y}
$$

and that

$$
\mathscr{H}^{c-m-t}\left(F_{y} ; \gamma_{y}^{*} \delta_{y}^{!} \mathbb{S}^{\bullet}\right)=\mathbb{H}^{c-m}\left(j_{V}^{!} f_{*} \mathbb{S}^{\bullet}[-t]\right)_{y} .
$$

(The second can also be derived from the first, duality, and the normal nonsingularity of $\gamma_{y}$.) It is then not hard to check that under these identifications the isomorphism

$$
\mathbb{H}^{c-m}\left(j_{V}^{*} f_{*} \mathbb{S}^{\bullet}[-t]\right)_{y} \cong \operatorname{Hom}_{\mathfrak{K}}\left(\mathbb{H}^{c-m}\left(j_{V} f_{*} \mathbb{S}^{\bullet}[-t]\right)_{y} ; \mathfrak{K}\right)
$$

of (4.3) agrees with the one induced by the self-duality of $f_{*} \mathbb{S}^{\bullet}[-t]$ of (4.1). The maps induced by the canonical morphisms

$$
j_{V} f_{*} \mathbb{S}^{\bullet}[-t] \rightarrow j_{V}^{*} f_{*} \mathbb{S}^{\bullet}[-t]
$$

and

$$
\gamma_{y}^{*} \delta_{y}^{!} \mathbb{S}^{\bullet} \rightarrow \gamma_{y}^{*} \delta_{y}^{*} \mathbb{S}^{\bullet}
$$

on $\mathbb{H}^{c-m}$ and $\mathscr{H}^{c-m-t}$, respectively, also agree. Thus

(4.4) Proposition. Let the hypothesis be as in (4.2). Let $F_{y}=f^{-1}(y)$. Then for $V$ a component of a stratum of $Y, \mathscr{P}_{V}\left(f_{*} \mathbb{S}^{\bullet}[-t]\right)_{y}$ is isomorphic to the nonsingular pairing induced by the duality isomorphism (4.3)

$$
\mathscr{H}^{c-m-t}\left(F_{y} ; \gamma_{y}^{*} \delta_{y}^{*} \mathbb{S}^{\bullet}\right) \cong \operatorname{Hom}_{\mathfrak{K}}\left(\mathscr{H}^{c-m-t}\left(F_{y} ; \gamma_{y}^{*} \delta_{y}^{!} \mathbb{S}^{\bullet}\right), \mathfrak{K}\right),
$$

on the image of the canonical map

$$
\mathscr{H}^{c-m-t}\left(F_{y} ; \gamma_{y}^{*} \delta_{y}^{!} \mathbb{S}^{\bullet}\right) \rightarrow \mathscr{H}^{c-m-t}\left(F_{y} ; \gamma_{y}^{*} \delta_{y}^{*} \mathbb{S}^{\bullet}\right)
$$


Let $L(y), y \in V$, be the link of the stratum $V$ of the Whitney stratified space $Y$ at the point $y$. Thus, $V$ has a closed tubular neighborhood $T_{V}$ in $Y$, for which there is a stratum-preserving locally trivial projection $\pi: T_{V} \rightarrow V$ with $\left(\pi^{-1}(y),\{y\}\right)$ stratum-preserving homeomorphic to the cone $(c L(y),\{c\})$. (See [GM3, I.1.4].) Let $N(y)=\pi^{-1}(y)$, the normal slice, with interior $\stackrel{\circ}{N}(y)$. Let

$$
F_{y}=f^{-1}(y) \stackrel{\omega_{y}}{\longrightarrow} f^{-1}(\stackrel{\circ}{N}(y)) \stackrel{\rho_{y}}{\longrightarrow} X
$$

be inclusion maps. By the first isotopy lemma applied to the composite

$$
f^{-1}\left(T_{V}\right) \stackrel{f \mid f^{-1} T_{V}}{\longrightarrow} T_{V} \stackrel{\pi}{\longrightarrow} V,
$$

$\rho_{y}$ is a normally nonsingular inclusion with trivial normal bundle. (This observation is due to MacPherson; see [GM3, I.1.6].)

Since $\rho_{y} \circ \omega_{y}=\gamma_{y} \circ \delta_{y}$,

$$
\omega_{y}^{*} \rho_{y}^{*} \mathbb{S}^{\bullet}=\gamma_{y}^{*} \delta_{y}^{*} \mathbb{S}^{\bullet}
$$

and

Hence we have

$$
\omega_{y}^{!} \rho_{y}^{*} \mathbb{S}^{\bullet}=\omega_{y}^{!} \rho_{y}^{!} \mathbb{S}^{\bullet}[m-2 c]=\gamma_{y}^{!} \delta_{y}^{!} \mathbb{S}^{\bullet}[m-2 c]=\gamma_{y}^{*} \delta_{y}^{!} \mathbb{S}^{\bullet}
$$

(4.5) Proposition. Let the hypotheses be as in (4.7). Let $F_{y}=f^{-1}(y)$. Then for $V$ a component of a stratum of $Y, \mathscr{P}_{V}\left(f_{*} \mathbb{S}^{\bullet}[-t]\right)_{y}$ is isomorphic to the nonsingular pairing induced by the duality isomorphism

$$
\mathscr{H}^{c-m-t}\left(F_{y} ; \omega_{y}^{*} \rho_{y}^{*} \mathbb{S}^{\bullet}\right) \cong \operatorname{Hom}_{\mathfrak{K}}\left(\mathscr{H}^{c-m-t}\left(F_{y} ; \omega_{y}^{!} \rho_{y}^{*} \mathbb{S}^{\bullet}\right), \mathfrak{K}\right),
$$

on the image of the canonically induced map

$$
\mathscr{H}^{c-m-t}\left(F_{y} ; \omega_{y}^{!} \rho_{y}^{*} \mathbb{S}^{\bullet}\right) \rightarrow \mathscr{H}^{c-m-t}\left(F_{y} ; \omega_{y}^{*} \rho_{y}^{*} \mathbb{S}^{\bullet}\right) .
$$

Using this result, we will reformulate (4.2). First,

$$
\rho_{y}^{!} \mathbb{S}^{\bullet}=\rho_{y}^{*} \mathbb{S}^{\bullet}[2 c-m]
$$

is a self-dual complex of sheaves on $K_{y}=f^{-1}(\stackrel{\circ}{N}(y))$. In fact,

$$
\mathfrak{D}\left(\rho_{y}^{!} \mathbb{S}^{\bullet}\right)[2 c+2 t] \cong \rho_{y}^{*} \mathfrak{D}\left(\mathbb{S}^{\bullet}\right)[2 c+2 t] \cong \rho_{y}^{*} \mathbb{S}^{\bullet}[2 c-m]=\rho_{y}^{!} \mathbb{S}^{\bullet} \text {. }
$$

This duality induces a perfect pairing

$$
\mathscr{Q}_{y}: \mathscr{H}_{c}^{-c-t}\left(K_{y} ; \rho_{y}^{!} \mathbb{S}^{\bullet}\right) \otimes \mathscr{H}^{-c-t}\left(K_{y} ; \rho_{y}^{!} \mathbb{S}^{\bullet}\right) \rightarrow \mathfrak{K},
$$

and hence an induced nonsingular pairing $\widehat{Q}_{y}$ on the image $\mathscr{F}_{y}$ of the natural map

$$
\mathscr{H}_{c}^{-c-t}\left(K_{y} ; \rho_{y}^{!} \mathbb{S}^{\bullet}\right) \rightarrow \mathscr{H}^{-c-t}\left(K_{y} ; \rho_{y}^{!} \mathbb{S}^{\bullet}\right)
$$

from hypercohomology with compact supports to hypercohomology. 
(4.6) Proposition. For $y \in V$,

$$
\left(\mathscr{J}_{y}, \widehat{\mathscr{Q}}_{y}\right) \cong\left(\mathscr{Z}^{V}\left(f_{*} \mathbb{S}^{\bullet}[-t]\right)_{y}, \mathscr{P}_{V}\left(f_{*} \mathbb{S}^{\bullet}[-t]_{y}\right)\right)
$$

Proof. By (4.5) and the equation $\rho_{y}^{!} \mathbb{S}^{\bullet}=\rho_{y}^{*} \mathbb{S}^{\bullet}[2 c-m], \mathscr{Z}^{V}\left(f_{*} \mathbb{S}^{\bullet}[-t]\right)_{y}$ is the image of the canonical map

$$
\mathscr{H}^{-c-t}\left(K_{y} ; \omega_{y_{*}} \omega_{y}^{!} \rho_{y}^{!} \mathbb{S}^{\bullet}\right) \rightarrow \mathscr{H}^{-c-t}\left(K_{y} ; \omega_{y_{*}} \omega_{y}^{*} \rho_{y}^{!} \mathbb{S}^{\bullet}\right),
$$

and $\mathscr{P}_{V}\left(f_{*} \mathbb{S}^{\bullet}[-t]\right)_{y}$ is induced by the duality pairing of the target and image obtained on hypercohomology from the isomorphism

$$
\mathfrak{D}\left(\omega_{y_{*}} \omega_{y}^{*} \rho_{y}^{!} \mathbb{S}^{\bullet}\right)[2 c+2 t]\left(\cong \omega_{y_{!}} \omega_{y}^{!} \mathfrak{D}\left(\rho_{y}^{!} \mathbb{S}^{\bullet}\right)[2 c+2 t]\right) \cong \omega_{y_{*}} \omega_{y}^{!} \rho_{y}^{!} \mathbb{S}^{\bullet}
$$

induced by the self-duality isomorphism of $\rho_{y}^{!} \mathbb{S}^{\bullet}$. Let $l_{y}$ be the inclusion of $K_{y}-F_{y}$ in $K_{y}$. Then we have the two distinguished triangles
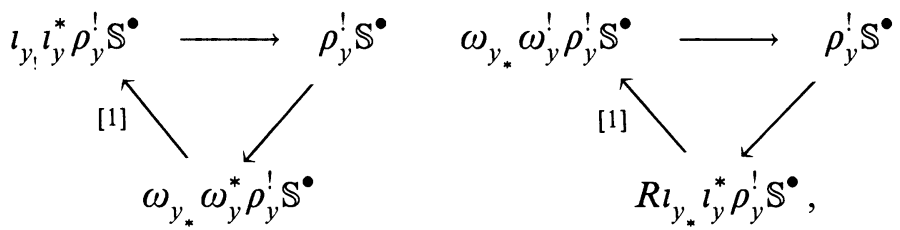

and these are dual to one another under isomorphisms induced by the duality isomorphism $\mathfrak{D}\left(\rho_{y}^{!} \mathbb{S}^{\bullet}\right)[2 c+2 t] \cong \rho_{y} \mathbb{S}^{\bullet}$.

By the first isotopy lemma applied to the composite

$$
K_{y}-F_{y}=f^{-1}(\stackrel{\circ}{N}(y)-\{y\}) \stackrel{f \mid}{\longrightarrow} N(y)-\{y\}=L(y) \times(0,1) \stackrel{\pi_{2}}{\longrightarrow}(0,1),
$$

$K_{y}-F_{y}$ is homeomorphic as a stratified space to the product

$$
f^{-1} L(y) \times(0,1) \text {. }
$$

Hence, by a standard argument using the hypercohomology spectral sequence,

$$
\mathscr{H}^{i}\left(l_{y_{1}} l_{y}^{*} \rho_{y}^{!} \mathbb{S}^{\bullet}\right)=\mathscr{H}_{c}^{i}\left(R l_{y}^{*} l_{y}^{*} \rho_{y}^{!} \mathbb{S}^{\bullet}\right)=0
$$

for all $i$. The result then follows upon application of hypercohomology to the first triangle and hypercohomology with compact supports to the second.

(4.7) Theorem. Let $f: X^{p} \rightarrow Y^{n}$ be a stratified map of oriented Whitney stratified spaces. Assume $X$ is compact, $Y$ has only even-codimension strata, and $t=\frac{1}{2}(p-n)$ is an integer. Let $\mathbb{S}^{\bullet}$ be a self-dual complex of sheaves on $X$. For $V$ a component of a stratum of $Y$ and $y \in V$, let $N(y)$ be the normal slice to $V$ at $Y$ and let $L(y)=\partial N(y)$ be the link. For $y$ in a singular stratum, let $E_{y}$ be the quotient

$$
E_{y}=f^{-1} N(y) / f^{-1} L(y) .
$$

For $y$ in a nonsingular stratum, let $E_{y}=f^{-1}(y)$. Let

$$
E_{y} \stackrel{i_{y}}{\longleftarrow} f^{-1} \stackrel{\circ}{N}(y)=K_{y} \stackrel{\rho_{y}}{\longrightarrow} X
$$


be inclusions. Let $Z_{y}=\left\{z_{y}\right\}=E_{y}-f^{-1} \stackrel{\circ}{N}(y)$. Then $\left(c=c(V)=\frac{1}{2} \operatorname{codim} V\right)$

(i) $\mathbb{S}^{\bullet}(y)=\tau_{\leq-c-t-1}^{Z_{y}} R i_{y_{*}} \rho_{y} \mathbb{S}^{\bullet}$ is a self-dual complex of sheaves on $E_{y}$, and

(ii) if $\mathfrak{H}_{f}^{V}\left(\mathbb{S}^{\bullet}\right)$ is the local system over $V$ with stalks

$$
\mathfrak{H}_{f}^{V}\left(\mathbb{S}^{\bullet}\right)_{y}=\mathscr{H}^{-c-t}\left(E_{y} ; \mathbb{S}^{\bullet}(y)\right)
$$

and with the induced nonsingular pairing $\mathscr{Q}_{\mathbb{S}^{\bullet}(y)}$ on each stalk, then $f_{*} \mathbb{S}^{\bullet}[-t]$ is cobordant to

$$
\sum_{\mathscr{V}} j_{*}\left(\mathbb{C}_{\bar{m}}^{\bullet}\left(\bar{V} ; \mathfrak{H}_{f}^{V}\left(\mathbb{S}^{\bullet}\right)\right)[c(V)]\right.
$$

(Note: $\tau_{\leq q}^{Z}$ denotes truncation over the closed set $Z$; see [GM2, 1.14]. For $y$ in a nonsingular stratum, $\mathbb{S}^{\bullet}(y)=\rho_{y}^{!} \mathbb{S}^{\bullet}$.)

A local system on $V$ is determined by its stalk and the action of $\pi_{1} V$ at one point. Hence we may also write the above sum as

$$
\sum_{\mathscr{V}} j_{*}\left(\mathbb{I} \mathbb{C}_{\bar{m}}^{\bullet}\left(\bar{V} ; \mathscr{H}^{-c-t}\left(E_{y_{V}} ; \mathbb{S}^{\bullet}\left(y_{V}\right)\right)\right)[c(V)]\right.
$$

$y_{V}$ is a chosen basepoint of $V$. With this notation we have

(4.8) Corollary. Let $f$ be as in (4.7), let $\mathfrak{L}$ be a local system of finitely generated $\mathfrak{K}$-modules over $X-X_{p-2}$, with a pairing

$$
\mathfrak{L} \otimes_{\mathfrak{K}} \mathfrak{L} \rightarrow \mathfrak{K}
$$

that is nonsingular on stalks. Then $f_{*} \mathbb{I} \mathbb{C}_{\bar{m}}^{\bullet}(X ; \mathfrak{L})[-t]$ is cobordant to

$$
\sum_{\mathscr{V}} j_{*} \mathbb{I} \mathbb{C}_{\bar{m}}^{\bullet}\left(\bar{V} ; I H_{c+t}^{\bar{m}}\left(E_{y_{V}} ; \mathfrak{L}\right)\right)[c(V)] .
$$

Proof. By [GM2, 5.4.1],

$$
\rho_{y}^{!} \mathbb{I} \mathbb{C}_{\bar{m}}^{\bullet}(X ; \mathfrak{L})=\mathbb{I} \mathbb{C}_{\bar{m}}^{\bullet}\left(f^{-1} \stackrel{\circ}{N}(y) ; \mathfrak{L}\right) .
$$

By the Deligne construction for the intersection complex [GM2, §3],

$$
\tau_{\leq-c-t-1} R i_{y_{*}} \mathbb{I} \mathbb{C}_{\bar{m}}^{\bullet}\left(f^{-1} \stackrel{\circ}{N}(y) ; \mathfrak{L}\right)=\mathbb{I} \mathbb{C}_{\bar{m}}^{\bullet}\left(E_{y} ; \mathfrak{L}\right) .
$$

By [2.3], this has the following immediate consequence, where the symbol " $\sim$ " denotes equivalence of modules with symmetric forms in the Witt group $\mathscr{W}(\mathfrak{K})$ of such forms.

(4.9) Corollary. Let $f$ be as in (4.7) and $\mathfrak{L}$ as in (4.8). Let $p$ be even. Then, in the Witt group $\mathscr{W}(\mathfrak{K})$,

$$
I H_{p / 2}^{\bar{m}}(X ; \mathfrak{L}) \sim \sum_{\mathscr{V}} I H_{n / 2-c}^{\bar{m}}\left(\bar{V} ; I H_{c+t}^{\bar{m}}\left(E_{y_{V}} ; \mathfrak{L}\right)\right) .
$$

Proof of (4.7). By [GM2, 9.1] (or by (1.3)), there exists a unique isomorphism

$$
\mathfrak{D}\left(\mathbb{S}^{\bullet}(y)\right)[2 c+2 t] \cong \mathbb{S}^{\bullet}(y),
$$


in the derived category, extending the duality isomorphism

$$
\mathfrak{D}\left(\rho_{y}^{!} \mathbb{S}^{\bullet}\right)[2 c+2 t] \cong \rho_{y}^{*} \mathfrak{D}\left(\mathbb{S}^{\bullet}\right)[2 c+2 t] \cong \rho_{y}^{*} \mathbb{S}^{\bullet}[2 c-m]=\rho_{y}^{!} \mathbb{S}^{\bullet}
$$

of $\rho_{y} ! \mathbb{S}^{\bullet}$ induced by that of $\mathbb{S}^{\bullet}$. Hence, by (4.6), it suffices to see that the natural morphisms

$$
R i_{y_{1}} \rho_{y}^{!} \mathbb{S}^{\bullet}=\tau_{\leq-N} R i_{y_{*}} \rho_{y}^{!} \mathbb{S}^{\bullet} \rightarrow \tau_{\leq-c-t} R i_{y_{*}} \rho_{y}^{!} \mathbb{S}^{\bullet},
$$

$N$ large, and

$$
\tau_{\leq-c-t} R i_{y_{*}} \rho_{y}^{!} \mathbb{S}^{\bullet} \rightarrow R i_{y_{*}} \rho_{y^{\prime}} \mathbb{S}^{\bullet}
$$

induce a surjection and an injection, respectively, on hypercohomology in dimension $-c-t$. For example, consider a distinguished triangle

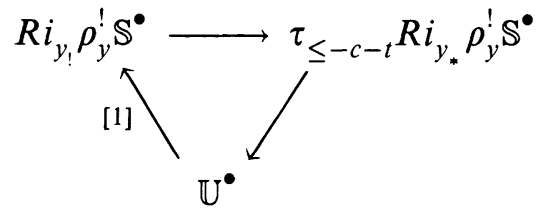

Then $\mathbb{U}^{\bullet}$ is supported on $\left\{z_{y}\right\}$ and $\mathbb{H}^{i} \mathbb{U}_{z_{y}}^{\bullet}=0$ for $i \geq-c-t$. Hence $\mathscr{H}^{i}\left(E_{y} ; \mathbb{U}^{\bullet}\right)$ vanishes for $i \geq-c-t$; in particular,

$$
\mathscr{H}^{-c-t}\left(E_{y} ; R i_{y_{!}} \rho_{y}^{!} \mathbb{S}^{\bullet}\right) \rightarrow \mathscr{H}^{-c-t}\left(E_{y} ; \tau_{\leq-c-t} R i_{*} \rho_{y} \mathbb{S}^{\bullet}\right)
$$

is a surjection. A similar argument shows that

$$
\mathscr{H}^{-c-t}\left(E_{y} ; \tau_{\leq-c-t} R i_{y_{*}} \rho_{y}^{!} \mathbb{S}^{\bullet}\right) \rightarrow \mathscr{H}^{-c-t}\left(E_{y} ; R i_{*} \rho_{y} \mathbb{S}^{\bullet}\right) \text {, }
$$

is an injection.

\section{Characteristic Classes}

Let $Y^{m}$ be a compact oriented stratified pseudomanifold, and let $\mathbb{S}^{\bullet} \in$ $D^{b}(Y ; \mathfrak{K})$ be a symmetric or skew symmetric complex of sheaves. Assume that $\mathfrak{K}$ is a subfield of the real numbers. For $m=2 n$, let the integer $\sigma\left(\mathbb{S}^{\bullet}\right)$ be the signature of the pairing

$$
\mathscr{Q}_{\mathbb{S}^{\bullet}}: \mathscr{H}^{-n}\left(Y ; \mathbb{S}^{\bullet}\right) \otimes_{\mathfrak{K}} \mathscr{H}^{-n}\left(Y ; \mathbb{S}^{\bullet}\right) \rightarrow \mathfrak{K} .
$$

(By definition, the signature of a skew pairing is trivial, whereas the signature of a symmetric pairing is the number of positive entries less the number of negative ones in a diagonalization over the real numbers.) For $m$ odd, we set

$$
\sigma\left(\mathbb{S}^{\bullet}\right)=0 \text {. }
$$

Let $W$ be an oriented, stratified pseudomanifold, and let

$$
\alpha: W^{w} \rightarrow Y^{m}
$$

be a normally nonsingular embedding [GM2, §5]. Then

$$
\mathfrak{D}\left(\alpha^{!} \mathbb{S}^{\bullet}\right)[w] \cong \alpha^{*} \mathfrak{D}\left(\mathbb{S}^{\bullet}\right)[w] \cong \alpha^{*} \mathbb{S}^{\bullet}[w-m] \cong \alpha^{!} \mathbb{S}^{\bullet},
$$


i.e., $\alpha ! \mathbb{S}^{\bullet}$ is a self-dual complex of sheaves over $V$. There is also a map

$$
\alpha^{\prime}: H_{i}(Y ; \mathbb{Q}) \rightarrow H_{i-m+w}(Y ; \mathbb{Q})
$$

given geometrically by intersection of cycles with $V$ or by the Thom isomorphism, and algebraically by

$$
\alpha^{*} \mathbb{D}_{Y}^{\bullet} \cong \alpha^{\prime} \mathbb{D}_{Y}^{\bullet}[m-w]=\mathbb{D}_{W}^{\bullet}[m-w],
$$

$\mathbb{D}_{Y}^{\bullet}$ the Verdier dualizing complex.

(5.1) Theorem. There exist unique classes $L_{i}\left(\mathbb{S}^{\bullet}\right) \in H_{i}(Y ; \mathbb{Q})$, defined for selfdual complexes of sheaves over stratified pseudomanifolds, with the following properties:

(i) $L_{0}\left(\mathbb{S}^{\bullet}\right)=\sigma\left(\mathbb{S}^{\bullet}\right) \in H_{0}(Y)=\mathbb{Z}, Y$ connected, and

(ii) if $\alpha: W^{w} \rightarrow Y^{m}$ is a normally nonsingular inclusion with trivial normal bundle, then

$$
L_{i-m+w}\left(\alpha \mathbb{S}^{\bullet}\right)=\alpha^{!} L_{1}\left(\mathbb{S}^{\bullet}\right)
$$

This result is actually an immediate consequence of the thethods of [CSW], from which the topological invariance of these classes also follows. For $Y$ with even-codimension strata and $\mathbb{S}^{\bullet}=\mathbb{I} \mathbb{C}_{\bar{m}}^{\bullet}(Y ; \mathbb{Q})$ the intersection complex, these classes are the homology $L$-classes $L_{i}(Y)$ of [GM1], and (4.1) can also be proven along the same lines, at least if $Y$ is a p.l. space. (However, homeomorphism invariance is not immediately clear in this approach.) The conditions (i) and (ii) are actually equivalent to

(iii) if $\alpha: W^{w} \rightarrow Y^{m}$ is a normally nonsingular inclusion with trivial normal bundle, then

$$
\sigma\left(\alpha ! \mathbb{S}^{\bullet}\right)=\alpha^{!} L_{m-w}\left(\mathbb{S}^{\bullet}\right)
$$

It is not hard to see that

$$
\alpha^{!} L_{m-w}\left(\mathbb{S}^{\bullet}\right)=\left\langle g^{*} l, L_{m-w}\left(\mathbb{S}^{\bullet}\right)\right\rangle,
$$

where $g: Y \rightarrow S^{m-v}$ is obtained from the Thom-Pontrjagin construction applied to a trivialization of the normal bundle of $W$ and $l \in H^{m-w}\left(S^{m-w}\right)$ is the appropriate generator. The uniqueness in (4.1) follows from the fact that a nonzero multiple of every cohomology class can be stably represented as $g^{*} l$ and the usual transversality arguments.

For $n-i$ odd, $L_{i}\left(\mathbb{S}^{\bullet}\right)=0$. For $Y$ with connected nonsingular part,

$$
L_{m}\left(\mathbb{S}^{\bullet}\right)=\sigma\left(\mathscr{P}^{Y}\left(\mathbb{S}^{\bullet}\right)_{y}\right)[Y],
$$

$[Y]$ is the orientation class, $y$ a nonsingular point.

(5.2) Proposition. Let $\mathbb{S}^{\bullet}$ and $\mathbb{S}_{1}^{\bullet}$ be cobordant self-dual complexes of sheaves on $Y$, over $\mathfrak{K}$. Then $L_{i}\left(\mathbb{S}^{\bullet}\right)=L_{i}\left(\mathbb{S}_{1}^{\bullet}\right)$.

Proof. Let $\alpha: W^{w} \rightarrow Y^{m}$ be the inclusion of a normally nonsingular stratified pseudomanifold. Then, from the isomorphism $\alpha^{*} \mathbb{A}^{\bullet} \cong \alpha^{!} \mathbb{A}[m-v]$ and the 
definition of cobordism, it is not hard to see that $\alpha ! \mathbb{S}^{\bullet}$ and $\alpha \cdot \mathbb{S}_{1}^{\bullet}$ are also cobordant. Hence, by (2.3) and the fact that the signature is an invariant of the Witt group,

$$
\sigma\left(\alpha \mathbb{S}^{\bullet}\right)=\sigma\left(\alpha ! \mathbb{S}_{1}^{\bullet}\right)
$$

Hence, by the uniqueness of these classes satisfying (iii), they must agree.

(5.3) Proposition. Let $V$ be a component of the stratum of codimension $2 c$ of the stratified pseudomanifold $Y$, assumed to have only even-codimension strata. Let $\mathbb{S}^{\bullet}$ be a self-dual complex of sheaves on $\bar{V}$, and let $j$ be the inclusion $\bar{V} \subset Y$. Then $j_{*} \mathbb{S}^{\bullet}[c]$ is a self-dual complex of sheaves on $Y$ and

Proof. Left to the reader.

$$
L_{i}\left(j_{*} \mathbb{S}^{\bullet}[c]\right)=j_{*} L_{i}\left(\mathbb{S}^{\bullet}\right) \text {. }
$$

(5.4) Proposition. Let $\mathbb{S}_{1}^{\bullet}$ and $\mathbb{S}_{2}^{\bullet}$ be self-dual complexes of sheaves on $Y$. Then

Proof. Obvious.

$$
L_{i}\left(\mathbb{S}_{1}^{\bullet} \oplus \mathbb{S}_{2}^{\bullet}\right)=L_{i}\left(\mathbb{S}_{1}^{\bullet}\right)+L_{i}\left(\mathbb{S}_{2}^{\bullet}\right)
$$

(5.5) Proposition. Let $f: X^{p} \rightarrow Y^{m}$ be a stratified map of compact oriented Whitney stratified pseudomanifolds. Let $\mathbb{S}^{\bullet}$ be a self-dual complex of sheaves on $X$. Assume that $p-m$ is even and let $t=\frac{1}{2}(p-m)$. Then

$$
L_{i}\left(f_{*} \mathbb{S}^{\bullet}[-t]\right)=f_{*} L_{i}\left(\mathbb{S}^{\bullet}\right) \text {. }
$$

Proof. Clearly, for $m=2 n$ and $p=2 q$,

$$
\mathscr{H}^{-n}\left(Y ; f_{*} \mathbb{S}^{\bullet}[-t]\right) \cong \mathscr{H}^{-q}\left(X ; \mathbb{S}^{\bullet}\right)
$$

and

In particular,

$$
\mathscr{Q}_{f_{*} \mathbb{S}^{\bullet}[-t]}=\mathscr{Q}_{\mathbb{S}^{\bullet}}
$$

and hence

$$
\sigma\left(f_{*} \mathbb{S}^{\bullet}[-t]\right)=\sigma\left(\mathbb{S}^{\bullet}\right)
$$

$$
f_{*} L_{0}\left(\mathbb{S}^{\bullet}\right)=L_{0}\left(f_{*} \mathbb{S}^{\bullet}[-t]\right)
$$

Let $W \rightarrow Y$ be the inclusion of a normally nonsingular stratified pseudomanifold, with trivial normal bundle. By transversality applied to the result of the Thom-Pontrjagin construction, or by a stratumwise smoothing theory argument, it may be assumed that $V$ is Whitney stratified with smooth trivial normal bundle. An application of Thom's first isotopy lemma to the submersion

$$
f^{-1}\left(W \times \mathbb{R}^{m-w}\right) \rightarrow W \times \mathbb{R}^{m-w} \stackrel{\pi_{2}}{\longrightarrow} \mathbb{R}^{m-w}
$$

shows that the inclusion

$$
\beta: f^{-1}(W) \rightarrow Y
$$

is also a normally nonsingular embedding of stratified pseudomanifold in $X$. Hence

$$
L_{0}\left(\beta^{!} \mathbb{S}^{\bullet}\right)=\beta^{!} L_{m-w}\left(\mathbb{S}^{\bullet}\right) .
$$


Let

$$
h=f \mid f^{-1}(W): f^{-1} W \rightarrow W
$$

Then

$$
h_{*} \beta^{!} \mathbb{S}^{\bullet}[-t]=\alpha ! f_{*} \mathbb{S}^{\bullet}[-t]
$$

as self-dual complexes of sheaves, and the equation

$$
h_{*} \circ \beta^{!}=\alpha ! \circ f_{*}
$$

also holds on homology. Hence

$$
\begin{aligned}
\alpha^{!} f_{*} L_{m-w}\left(\mathbb{S}^{\bullet}\right) & =h_{*} \beta^{!} L_{m-w}\left(\mathbb{S}^{\bullet}\right)=h_{*}\left(L_{0}\left(\beta^{!} \mathbb{S}^{\bullet}\right)\right) \\
& =L_{0}\left(h_{*} \beta^{!} \mathbb{S}^{\bullet}[-t]\right)=L_{0}\left(\alpha^{!} f_{*} \mathbb{S}^{\bullet}[-t]\right) .
\end{aligned}
$$

Hence, by the uniqueness statement in (4.1),

$$
f_{*} L_{i}\left(\mathbb{S}^{\bullet}\right)=L_{i}\left(f_{*} \mathbb{S}^{\bullet}[-t]\right)
$$

Combining the previous results with (4.7) we obtain

(5.6) Theorem. Let $f: X^{p} \rightarrow Y^{m}$ be a stratified map of compact oriented Whitney stratified pseudomanifolds. Let $\mathbb{S}^{\bullet}$ be a self-dual complex of sheaves on $X$. Assume that $p-m$ is even and let $t=\frac{1}{2}(p-m)$. Assume $Y$ has only evencodimension strata. Then

$$
f_{*} L_{i}\left(\mathbb{S}^{\bullet}\right)=\sum_{\mathscr{V}} j_{*} L_{i}\left(\mathbb{I} \mathbb{C}_{\bar{m}}^{\bullet}\left(\bar{V} ; \mathfrak{H}_{f}^{V}\left(\mathbb{S}^{\bullet}\right)\right)\right)
$$

$\mathscr{V}$ the set of components of strata of $Y$.

Note. In the above formula, the terms may be written in the notation of [CS2, §6] as twisted $L$-classes

$$
L_{i}\left(\mathbb{I} \mathbb{C}_{\bar{m}}^{\bullet}\left(\bar{V} ; \mathscr{H}^{-c(V)-t}\left(E_{y_{V}} ; \mathbb{S}^{\bullet}\left(y_{V}\right)\right)\right)\right)=L_{i}^{\mathscr{Q}\left(\mathbb{S}^{\bullet}\left(y_{V}\right)\right)}(\bar{V}),
$$

associated to the pairing $\mathscr{Q}\left(\mathbb{S}^{\bullet}\left(y_{V}\right)\right)$ induced on $\mathscr{H}^{-c-t}\left(E_{y_{V}} ; \mathbb{S}^{\bullet}\left(y_{V}\right)\right)$ be the self-duality of $\mathbb{S}^{\bullet}\left(y_{V}\right), y_{V}$ a basepoint of $V$ as in the discussion following (4.7). Thus (5.6) becomes the statement

$$
L_{i}\left(f_{*} \mathbb{S}^{\bullet}\right)=\sum_{\mathscr{V}} j_{*} L_{i}^{\mathscr{Q}\left(\mathbb{S}^{\bullet}\left(y_{V}\right)\right)}(\bar{V}) .
$$

If the strata $V$ are simply connected, or, more generally, the local systems $\mathfrak{H}_{f}^{V}\left(\mathbb{S}^{\bullet}\right)$ are trivial, then it is not hard to see from $(4.1)$ that

$$
L_{i}^{\mathscr{Q}\left(\mathbb{S}^{\bullet}\left(y_{V}\right)\right)}=\sigma\left(\mathscr{Q}\left(\mathbb{S}^{\bullet}\left(y_{V}\right)\right)\right) L_{i}(\bar{V}),
$$

where the integer $\sigma\left(\mathscr{Q}\left(\mathbb{S}^{\bullet}\left(y_{V}\right)\right)\right.$ is the signature of this pairing. Then the formula in $(5.6)$ reduces to

$$
L_{i}\left(f_{*} \mathbb{S}^{\bullet}\right)=\sum_{\mathscr{V}} \sigma\left(\mathscr{Q}\left(\mathbb{S}^{\bullet}\left(y_{V}\right)\right)\right) j_{*} L_{i}(V) .
$$


We conclude this section with an explicit statement for the case of the intersection complex, as in (4.7). For $Z$ an even-dimensional stratified pseudomanifold with even-codimension strata, we write $Q(Z)$ for $\mathscr{Q}\left(\mathbb{I} \mathbb{C}_{\bar{m}}^{\bullet}(Z ; \mathfrak{K})\right)$, the middledimensional nonsingular intersection pairing on intersection homology, with signature $\sigma(Z)$.

(5.7) Theorem. Let $f: X^{p} \rightarrow Y^{m}$ be a stratified map of compact oriented Whitney stratified pseudomanifolds. Let $\mathbb{S}^{\bullet}$ be a self-dual complex of sheaves on $X$. Assume that $p-m$ is even and let $t=\frac{1}{2}(p-m)$. Assume $Y$ has only evencodimension strata. Let $\mathscr{V}$ be the set of components of strata of $Y$, and choose $y_{V} \in V$. Let $N\left(y_{V}\right)$ be the normal slice to $V$ at $y$, and let $L(y)=\partial N(y)$ be the link. Let $E_{y}$ be the stratified pseudomanifold

$$
E_{y}=f^{-1} N(y) \cup_{f^{-1} L(y)} c\left(f^{-1} L(y)\right)
$$

be obtained from $f^{-1} N(y)$ by attaching the cone on $f^{-1} L(y)$, for $y$ in a singular stratum, and let

$$
E_{y}=f^{-1}(y)
$$

for $y$ in a nonsingular stratum. Let $L_{i}(X) \in H_{i}(X ; \mathbb{Q})$ be the (GoreskyMacPherson) L-classes. Then

$$
f_{*} L_{i}(X)=\sum_{\mathscr{V}} j_{*} L_{i}^{Q\left(E_{y_{V}}\right)}(\bar{V}),
$$

(5.8) Corollary. Assume that the local systems given by $I H_{c+t}^{\bar{m}}\left(E_{y_{V}}\right)$ are trivial. (For example, let all $V \in \mathscr{V}$ be simply connected.) Then

$$
f_{*} L_{i}(X)=\sum_{\mathscr{V}} \sigma\left(E_{y_{V}}\right) j_{*} L_{i}(\bar{V})
$$

\section{Applications}

In this section we briefly give a few simple applications. Some more elaborate applications will be discussed elsewhere.

(6.1) Mismatch of dimensions. It is a well-known result of Chern-HirzebruchSerre [CHS] that the signature of the total space of a fibre bundle over a simply connected base of dimension not congruent to zero modulo four is zero. We extend this result to stratified maps.

Theorem. Let $f: X \rightarrow Y$ be a stratified map of oriented even-dimensional Whitney stratified spaces. Assume that each stratum of $Y$ is simply connected and has dimension congruent to two modulo four. Then

$$
\sigma(X)=0 \text {. }
$$

Note that this result is new even in the case that $X$ and $Y$ are homeomorphic to manifolds (but their stratification exhibiting $f$ as a stratified map is nontrivial). 
Proof. By (5.8),

$$
\sigma(X)=L_{0}(X)=f_{*} L_{0}(X)=\sum_{\mathscr{V}} \sigma\left(E_{y_{V}}\right) j_{*} L_{0}(\bar{V})=\sum_{\mathscr{V}} \sigma\left(E_{y_{V}}\right) \sigma(\bar{V}) .
$$

Since $\bar{V}$ (and $E_{y_{V}}$ as well) has dimension congruent to two $\bmod 4$, the last term is zero.

This result remains valid as long as for each $V \in \mathscr{V}$, the monodromy action of $\pi_{1} V$ on the middle-dimensional intersection homology of $E_{y_{V}}$ is trivial.

(6.2) Resolutions, $L$-classes, and projective varieties. Let $Y$ be a compact Whitney stratified space with only even-codimension strata. Let $\bar{p}$ be a perversity. Then a resolution of $Y$ of size at most $\bar{p}$ will be defined as a stratified map $f: X \rightarrow Y$ satisfying the following:

(i) $f \mid f^{-1}\left(Y-\Sigma_{Y}\right): f^{-1}\left(Y-\Sigma_{Y}\right) \rightarrow Y-\Sigma_{Y}$ is a homeomorphism;

(ii) $X$ is homeomorphic to a compact manifold; and

(iii) for $y$ in a stratum of $Y$ of codimension $2 c, \operatorname{dim} f^{-1}(y) \leq p(c)$.

For example, a resolution of size $\bar{m}$ of a stratified space with even-codimension strata is a small resolution, and a resolution of size $\bar{l}$, the logarithmic perversity, is a semismall resolution.

Theorem. Let $Y$ be a compact oriented Whitney stratified space with oriented even-codimension strata. Assume that for $V$ a component of a stratum of $Y$, $\bar{V}$ has a resolution of size at most $\bar{p}_{V}$. Then $L_{i}(Y)$ is a sum of classes in the images of the maps

$$
I H_{i}^{\bar{p}_{V}}(\bar{V} ; \mathbb{Q}) \rightarrow H_{i}(\bar{V} ; \mathbb{Q}) \rightarrow H_{i}(Y ; \mathbb{Q}) .
$$

The obvious consequence for high-dimensional $L$-classes is valid under weaker hypotheses.

Theorem. Let $Y$ be a compact oriented Whitney stratified space with oriented even-codimension strata. Assume $Y$ has a resolution of size at most $\bar{p}$. Then, for $i>\operatorname{dim} \Sigma_{Y}, \Sigma_{Y}$ the union of the singular strata, $L_{i}(Y)$ is in the image of the natural map

$$
I H_{i}^{\bar{p}}(Y ; \mathbb{Q}) \rightarrow H_{i}(Y ; \mathbb{Q}) .
$$

These results can be viewed as obstructions to the existence of resolutions of at most a given size. For example, we have

Corollary. Let $Y$ be as in the previous theorem, and let $L_{Y}$ be the boundary of a regular neighborhood of $\Sigma_{Y}$. Let

$$
\mathscr{L}_{i}\left(L_{Y}\right) \in H^{i}(L ; \mathbb{Q})
$$

be the Thom-Hirzebruch cohomology L-classes of the manifold $L_{Y}$. Then

$$
\mathscr{L}_{i}\left(L_{Y}\right)=0
$$


for

$$
\operatorname{dim} \Sigma_{Y}+p\left(\operatorname{codim} \Sigma_{Y}\right)<i<\operatorname{codim} \Sigma_{Y}
$$

For projective algebraic varieties, these results can be strengthened to the middle perversity. (For local complete intersections and the perversity $\bar{l}$, the conclusions of the above results already follow from [GM2, 5.6.3].)

Theorem. Let $Y$ be a projective algebraic variety. Then, for $i>\operatorname{dim} \Sigma_{Y}, \Sigma_{Y}$ the union of the singular strata, $L_{i}(Y)$ is in the image of the natural map

$$
I H_{i}^{\bar{m}}(Y ; \mathbb{Q}) \rightarrow H_{i}(Y ; \mathbb{Q}) \text {. }
$$

Corollary. Let $Y$ be a projective algebraic variety, and let $L_{Y}$ be the boundary of a regular neighborhood of the singular set. Then

$$
\mathscr{L}_{i}\left(L_{Y}\right)=0
$$

for

$$
\frac{1}{2}\left(\operatorname{dim} Y+\operatorname{dim} \Sigma_{Y}\right) \leq i<\operatorname{codim} \Sigma_{Y} .
$$

(In this result $\operatorname{dim} Y$ is the real dimension of $Y$.)

Similarly, in the algebraic case the first theorem can be improved to the middle perversity of the strata.

Theorem. Let $Y$ be a projective algebraic variety, stratified so that the closure of each component of each stratum has a resolution that is a stratified map with respect to the given stratification. Then $L_{i}(Y)$ is a sum of classes in the images of the maps

$$
I H_{i}^{\bar{m}}(\bar{V} ; \mathbb{Q}) \rightarrow H_{i}(\bar{V} ; \mathbb{Q}) \rightarrow H_{i}(Y ; \mathbb{Q}) .
$$

The first result is proved by induction on dimension. For simplicity we discuss only the case where all components of strata are simply connected. Then

$$
f_{*} L_{i}(X)=\sum_{\mathscr{V}} \sigma\left(E_{y_{V}}\right) j_{*} L_{i}(\bar{V}),
$$

for $f: X \rightarrow Y$ a resolution of size at most $\bar{p}$. By induction, it suffices to prove that $f_{*} L_{i}(X)$ is in the image of the natural map

$$
I H_{i}^{\bar{p}}(Y ; \mathbb{Q}) \rightarrow H_{i}(Y ; \mathbb{Q}) .
$$

By the same argument as [GM2, 6.3],

$$
R f_{*} \mathbb{Q}_{X}[\operatorname{dim} X]=R f_{*} \mathbb{D}_{X}^{\bullet} \cong \mathbb{I} \mathbb{C}_{\bar{p}}^{\bullet}(Y ; \mathbb{Q}) \text {. }
$$

Hence $f$ induces an isomorphism

$$
H_{i}(X ; \mathbb{Q}) \cong I H_{i}^{\bar{p}}(Y ; \mathbb{Q}) .
$$

(Alternate argument. Use chain representatives and apply transversality to inverse images of strata.) Hence $f_{*} L_{i}(X)$ comes from $I H_{i}^{\bar{p}}(Y ; \mathbb{Q})$. The final result above is proven similarly, using the consequence of the decomposition theorem $[\mathrm{BBD}]$ that $f$ induces a surjection of $H_{i}(X ; \mathbb{Q})$ onto $I H_{i}^{\bar{m}}(Y ; \mathbb{Q})$. 
The second and third theorem can be proven similarly. They also have more elementary proofs; we have included them here to indicate the nontriviality of the type of restriction being obtained.

Finally, we remark that the classes in intersection homology groups that lift the $L$-classes actually are well defined in terms of the resolutions. It would be interesting to know what aspects of the geometry of $Y$ they detect and, in particular, to what extent they depend on the choice of the resolution.

(6.3) Discriminants. Let $X$ be an oriented even-dimensional stratified space with even-codimension strata. Let $\mathfrak{K}$ be a field. Then the nonsingular pairing on middle-dimensional intersection homology has a well-defined discriminant

$$
d(X) \in \mathfrak{K}^{\times} /\left(\mathfrak{K}^{\times}\right)^{2},
$$

depending only upon the element in the Witt group represented by this pairing. Let $I \chi(X)$ denote the Euler characteristic of $X$ with respect to middle perversity intersection homology. Then from (4.7) one can deduce:

Theorem. Let $f: X \rightarrow Y$ be a stratified map of oriented even-dimensional Whitney stratified spaces with oriented even-codimension strata. Let $\mathscr{V}$ be the components of strata of $Y$, and let $y_{V} \in V$ be chosen, $V \in \mathscr{V}$. Let $E_{y_{V}}$ be obtained from $f^{-1} N\left(y_{V}\right)$ by coning off the boundary, $N\left(y_{V}\right)$ the normal slice to $V$ at $y_{V}$. Assume each $V$ in $\mathscr{V}$ is simply connected. Then

$$
d(X)=\prod_{\mathscr{V}} d(\bar{V})^{I \chi\left(E_{y_{V}}\right)} d\left(E_{y_{V}}\right)^{I \chi(\bar{V})} .
$$

(6.4) Higher signatures, symmetric signatures, and surgery obstructions. The methods of this paper can also be applied to the study of the (Novikov) higher signature and its image under the assembly map, the (Miscenko-Ranicki) symmetric signature. We briefly indicate some of the results. For $X^{n}$ a compact, stratified pseudomanifold with even-codimension strata, the intersection chain complex over $\mathbb{Q}$ provides an orientation

$$
[X]_{\mathbb{L}} \in H_{n}\left(X ; \mathbb{L}_{\mathbb{Q}}^{*}\right)
$$

of $X$ in the symmetric $L$-spectrum over $\mathbb{Q}$. Its image

$$
\sigma_{\mathbb{Q}}^{\pi}(X) \in H_{n}\left(B \pi ; \mathbb{L}_{\mathbb{Q}}^{*}\right),
$$

$\pi$ a group equipped with map $\pi_{1} X \rightarrow \pi$ (e.g., $\pi=\pi_{1} X$ ), is the higher signature. (The original higher signature is the image of this in homology under a Pontrjagin character.) The image

$$
\sigma_{\mathbb{Q}}^{*}(X) \in L^{n}(\mathbb{Q} \pi)
$$

of $\sigma_{\mathbb{Q}}^{\pi}(X)$ under the assembly is the symmetric signature over $\mathbb{Q}$. 
Theorem. Let $f: X \rightarrow Y$ be a stratified map of compact oriented Whitney stratified spaces with even-codimension strata, and assume $\operatorname{dim} X-\operatorname{dim} Y$ is even. Then, under an assumption of trivial monodromy,

$$
f_{*}[X]_{\mathbb{L}}=\sum_{\mathscr{V}} \sigma\left(E_{y_{V}}\right) j_{*}[\bar{V}]_{\mathbb{L}},
$$

and hence,

$$
\sigma_{\mathbb{Q}}^{\pi}(X)=\sum_{\mathscr{V}} \sigma\left(E_{y_{V}}\right) \sigma_{\mathbb{Q}}^{\pi}(\bar{V}) \quad \text { and } \quad \sigma_{\mathbb{Q}}^{*}(X)=\sum_{\mathscr{V}} \sigma\left(E_{y_{V}}\right) \sigma_{\mathbb{Q}}^{*}(\bar{V}) .
$$

In the case of a resolution of singularities, for example, the first formula describes the failure of the map to preserve the $\mathbb{L}$-theory orientations, in terms of the structure of the singularities. Similar statements can be made for $K$ theory, and the result on equivariant $K$-theory mentioned in the introduction can be obtained by application of the assembly map to an equivariant version.

In the case of nontrivial monodromy for the middle intersection homology of $E_{y_{V}}$ as a local system over $V$, the terms in each sum must be replaced by their twisted analogues.

Corollary. The image in $L^{n}\left(\mathbb{Q}\left[\pi_{1} N\right]\right)$ of the symmetric surgery obstruction $\sigma^{*}(f) \in L^{n}\left(\pi_{1} N\right)$ of a stratifiable degree one map $f: M \rightarrow N$ manifolds can be computed from the singular set as

$$
\sigma^{*}(f)=\sigma^{*}(M)-\sigma^{*}(N)=\sum_{\mathscr{V}} \sigma\left(E_{y_{V}}\right) \sigma^{*}(\bar{V})
$$

in the case of trivial monodromy and

$$
\sigma^{*}(f)=\sum_{\mathscr{V}_{s}} \sigma_{t}^{*}(\bar{V})
$$

in general.

Here $\sigma_{t}$ is the twisted symmetric signature associated to the local system on $V \in \mathscr{V}_{s}=$ components of singular strata, with coefficients in the middle intersection homology of $E_{y_{V}}$.

For Goresky-Siegel spaces (i.e., locally torsionfree pseudomanifolds), these formulas make sense and are true over $\mathbb{Z} \pi$. (They also hold with respect to visible $L$-theory.)

These results imply that one can almost compute the surgery obstruction of a stratifiable normal map from the singular set. For example, it follows from a result of $[\mathbf{R}]$ that for a stratifiable normal map $(f, b)$ of degree one with target $N, 8 \sigma(f, b)$ in the surgery obstruction group $L_{n}\left(\pi_{1} N\right)$ is determined by the above formulas. In particular, under trivial monodromy assumptions, strata not of codimension divisible by four make no contribution at least to $8 \sigma(f, b)$.

Exact calculations can probably also be obtained, based on a more refined notion of quadratic complexes of sheaves, at least for stratifications for which the closures of components of singular strata are Goresky-Siegel spaces. 


\section{REFERENCES}

[At] M. F. Atiyah, The signature of fibre-bundles, Global Analysis, Papers in honor of K. Kodaira (D. C. Spencer and S. Iyanayaga, eds.), Princeton Univ. Press, Princeton, NJ, 1969, pp. 73-84.

[BBD] A.A. Beilinson, J. Berstein, and P. Deligne, Faisceaux pervers, analyse et topologie sur les espaces singuliers, Asterisque 100 (1982), 1-171.

[Bo] A. Borel et al., Intersection cohomology, Progress in Math., vol. 50, Birkhauser-Verlag, Boston, 1984.

[Br] W. Browder, Surgery on simply connected manifolds, Springer, New York, 1972.

[CS1] S. E. Cappell and J. L. Shaneson, Characteristic classes, singular embeddings, and intersection homology, Proc. Nat. Acad. Sci. U.S.A. 84 (1987), 3954-3956.

[CS2] _- Singular spaces, characteristic classes, and intersection homology, Ann. of Math. (2) (to appear).

[CSW] S. E. Cappell, J. L. Shaneson, and S. Weinberger, Classes characteristiques topologiques pour les actions des groupes sur les espaces singuliers, C.R. Acad. Sci. Paris Sér. I Math. (to appear).

[Ch] J. Cheeger, On Hodge theory of Riemannian pseudomanifolds, Proc. Sympos. Pure Math., vol. 36, Amer. Math. Soc., Providence, RI, 1980, pp. 91-146.

[CHS] S. S. Chern, F. Hirzebruch, and J.-P. Serre, The index of a fibered manifold, Proc. Amer. Math. Soc. 8 (1957), 587-596.

[GM1] M. Goresky and R. D. MacPherson, Intersection homology theory, Topology 19 (1980), $135-162$.

[GM2] _ Intersection homology. II, Invent. Math. 72 (1983), 77-130.

[GM3] _ Stratified Morse theory. Ergeb. Math. Grenzgeb. (3), Springer-Verlag, New York, 1980.

[GS] M. Goresky and P. Siegel, Linking pairings on singular spaces, Comment. Math. Helv. 58 (1983), 96-110.

[H] F. Hirzebruch, Topological methods in algebraic geometry, 3rd ed., Grundlehren Math. Wiss., vol. 131, Springer-Verlag, New York, 1978.

[M] R. D. MacPherson, Global questions in the topology of singular spaces, Proc. Internat. Congr. Math. 1983, North-Holland, New York, pp. 213-235.

[S] P. Siegel, Witt spaces: A geometric cycle theory for KO-homology theory at odd primes, Amer. J. Math. 105 (1983), 1067-1105.

[R] A. Ranicki, Exact sequences in the algebraic theory of surgery, Princeton Univ. Press, Princeton, NJ, 1981.

[We] S. Weinberger, The topological classification of stratified spaces (to appear).

Courant Institute of Mathematics, 251 Mercer Street, New York, New York 10012

Department of Mathematics, University of Pennsylvania, Philadelphia, PennsylvaNIA 19104 